\title{
ESF Nachwuchsforschergruppe
} E PISA

Energieautarke, drahtlose piezoelektrische MEMS Sensoren und

Aktoren in der Medizintechnik und Industrie 4.0

\section{Abschlussbericht}

\section{HerausgeberIn:}

Simon Böttger | Julien Bucher | David Kriebel | Katja Meinel |

Dmytro Solonenko | Martin Stiebing | Chris Stöckel

\section{Professuren:}

Zentrum für Mikrotechnologien | Prof. Karla Hiller | Projektleiterin

Professur Innovationsforschung und Technologiemanagement | Prof. Stefan Hüsig

Professur Mikrosysteme und Medizintechnik | Prof. Jan Mehner

Professur für Mikrotechnologie | Prof. Thomas Otto

Professur Nanoelectronics Technologies | Prof. Stefan E. Schulz

Professur Werkstoffe und Zuverlässigkeit mikrotechnischer Systeme | Prof. Bernhard Wunderle

Professur Halbleiterphysik | Prof. Dietrich R. T. Zahn

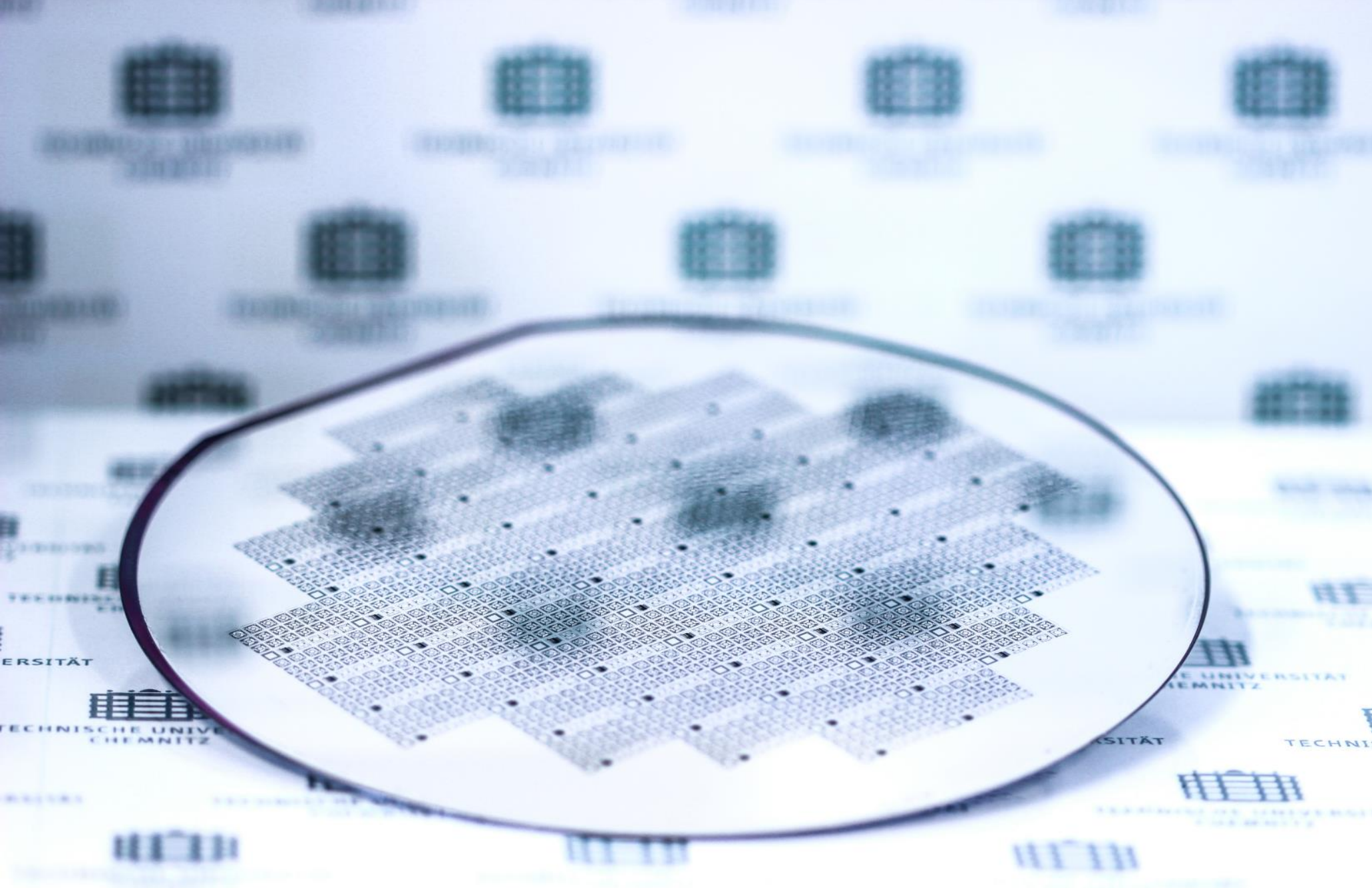





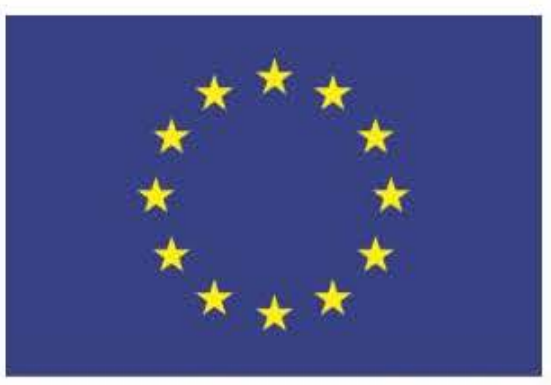

Europäische Union

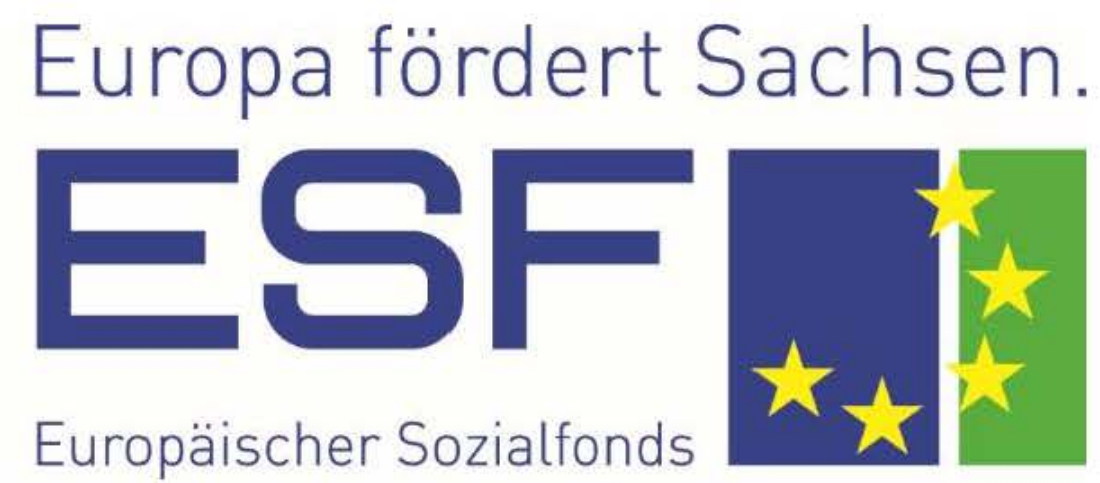

Europa fördert Sachsen. Europäischer Sozialfonds 


\section{Inhaltsverzeichnis}

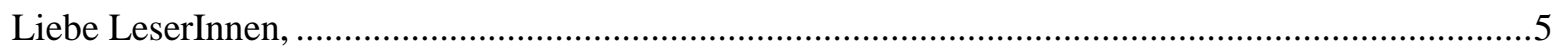

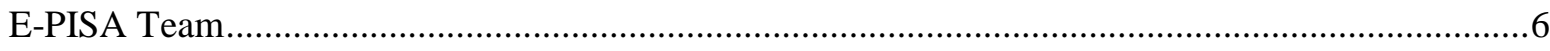

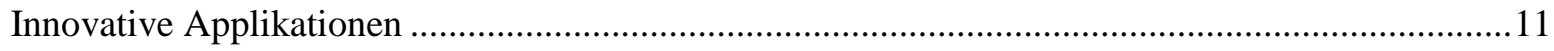

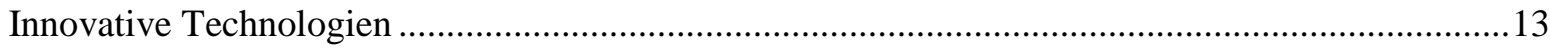

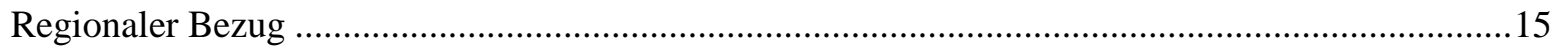

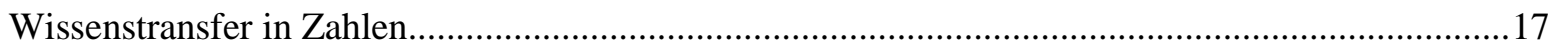

Piezoelektrisches Dünnschicht-Aluminiumnitrid für Mikrosysteme ................................................19

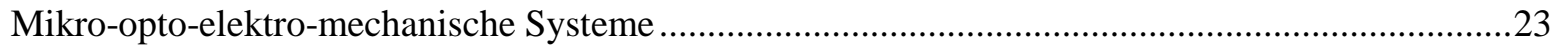

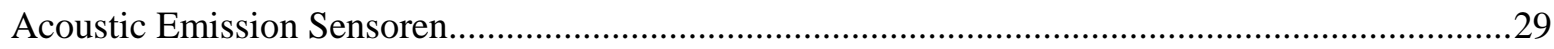

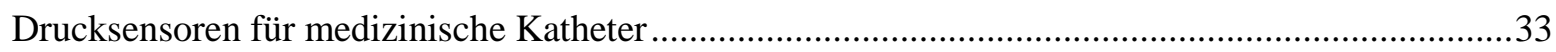

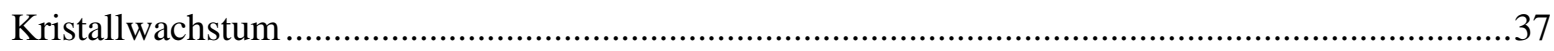

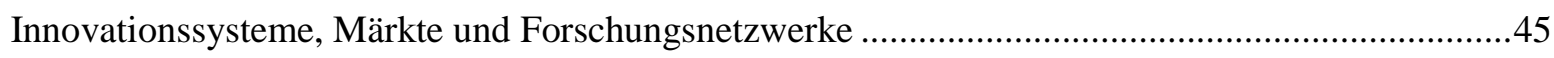

Zuverlässigkeit piezoelektrischer Schichtsysteme der Mikrotechnologie ........................................51

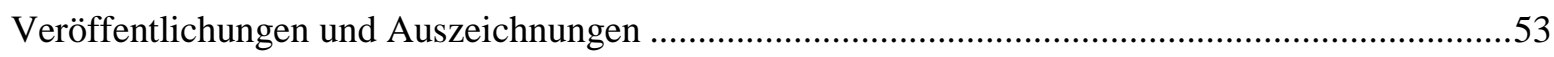

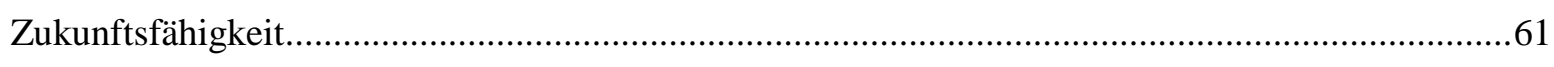

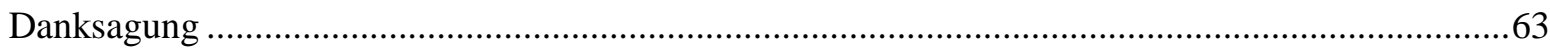




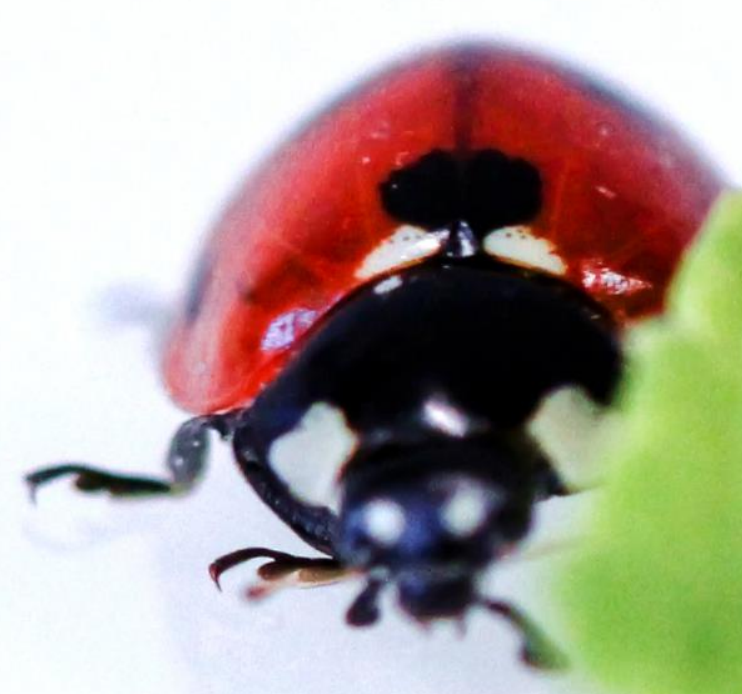


(हु) 
"The development of a new technology like microsystems is not only a technological step, but also a process of the society in correlations with many fields of our personal and social life."

Walter Lang

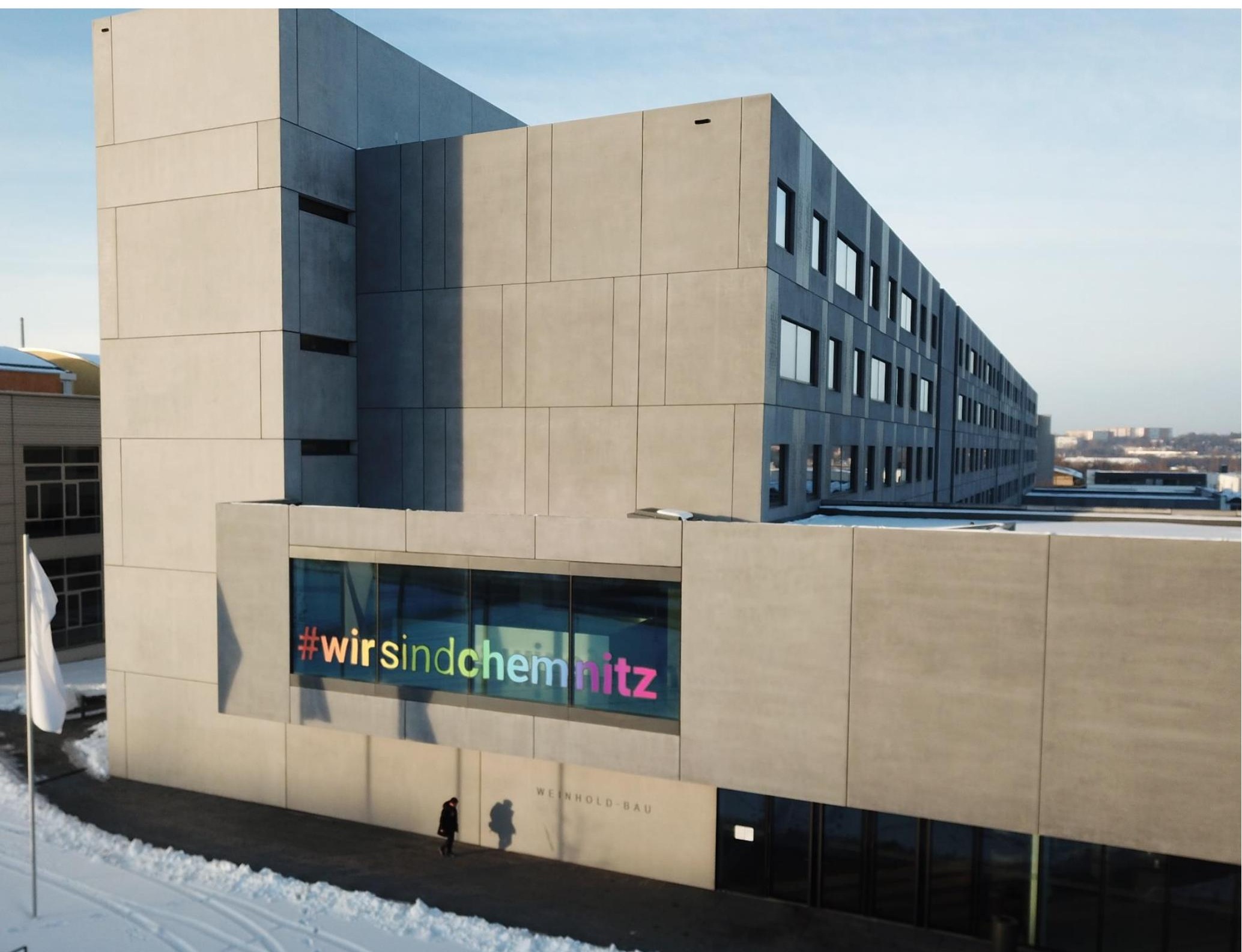




\section{Liebe LeserInnen,}

in dem ESF geförderten Projekt E-PISA sind verschiedene, hoch innovative, von der globalen Forschungslandschaft ausgezeichnete und gleichzeitig industrierelevante technische Entwicklungen im Bereich der Mikrosystemtechnik, Medizintechnik und Industrie 4.0 vorangetrieben worden.

Fokus der technischen Entwicklung waren die Grundlagenforschung und Applikationen von Mikrosystemen auf Basis piezoelektrischer Dünnschichten mit Aluminiumnitrid und Elektronik mit Carbon-Nano-Tubes.

Die im Projekt gewonnenen Erkenntnisse $\mathrm{zu}$ endoskopischen und hochfrequenten Mikrosystemen werden u.a. in Kooperation mit der Fraunhofer Gesellschaft und Industriepartnern in nachfolgender Entwicklung in medizintechnische Anwendungen überführt.

Die Forschung an Wake-Up MEMS wird ebenso in Überführungsprojekten mit Industriekunden im Bereich Industrie 4.0 und Medizintechnik anwenderspezifisch weiterentwickelt.

Des Weiteren wurden neue physikalische und experimentelle Grundlagen zu Zuverlässigkeit, Langzeitstabilität und Hochtemperaturverhalten der neuen Mikrotechnologien auf Basis des piezoelektrischen Dünnschicht-Aluminiumnitrids gewonnen. Diese Erkenntnisse können in zukünftiger Forschung in neue Applikationen überführt werden.

Während der Projektlaufzeit (2017-2020) der ESF geförderten Nachwuchsforschergruppe EPISA hat die TU Chemnitz aus gegebenem Anlass unter dem Hashtag \#Wirsindchemnitz ein klares Zeichen für Vielfalt und Weltoffenheit gesetzt. Wir, als Nachwuchsforschergruppe, sind stolz diese Grundsätze in unserer wissenschaftlichen Tätigkeit durch unsere Arbeits- und Lebensart vorleben zu können. Darüber hinaus gibt uns das Wirken als Wissenschaftler die Möglichkeit in zahlreichen Veröffentlichungen, neuen Kooperationen und auf weltweiten Konferenzen durch innovative und kreative Forschungsarbeit die Außenwirkung der Stadt Chemnitz und der Region Sachsen als High-Tech Standort zu vermitteln.

2020, im finalen Jahr des E-PISA-Projektes, wurde die Gesellschaft durch den Ausbruch des Corona-Virus erschüttert. Die WissenschaftlerInnen der Nachwuchsforschergruppe verstehen sich in der Pflicht durch ihre Forschung neue Impuls für die Medizintechnik zu setzen und den Anschub von Innovation für die Wirtschaft zu geben. 


\section{E-PISA Team}

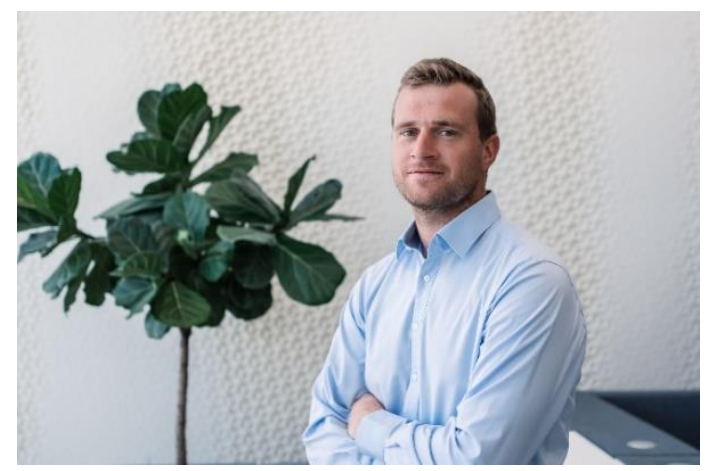

M.Sc. Simon Böttger

Wissenschaftler |

Fachgebiet CNT Heterosystemintegration

Simon Böttger war in der ESF Nachwuchsforschergruppe für die Heterosystemintegration von CNT-FETs in AlN-MOEMS verantwortlich. Dazu entwickelte er neuartige Methoden und Prozesse für die CNTChiralitätsseparation und CNT-Selbstassemblierung zur Anwendung auf bis zu 200mm-Wafer. Diese methodischen Entwicklungen mündeten in die Planung und den Aufbau von zwei Anlagen, welche inzwischen auch in anderen Forschungsprojekten am Standort Chemnitz eingesetzt werden.

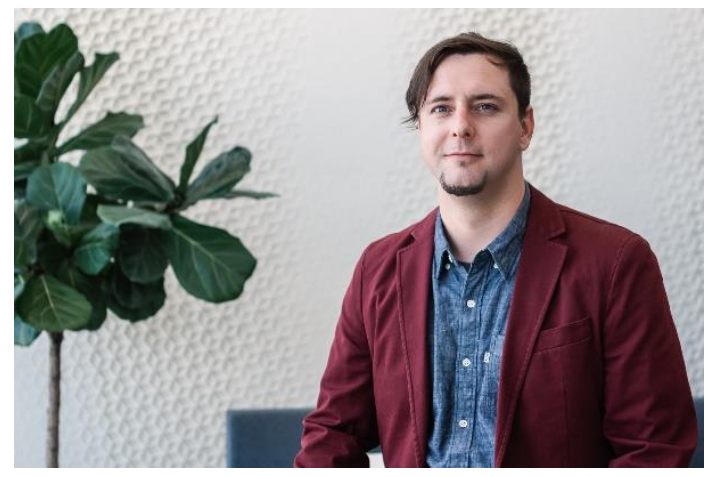

\section{Dr. Julien Bucher}

Stellv. Leiter Nachwuchsforschergruppe |

Fachgebiet Innovations- \& Technologie-management

Julien Bucher war stellvertretender Leiter der Nachwuchsforschergruppe und zudem für die Öffentlichkeitsarbeit zuständig. In seiner Forschung hat er sich mit der Evaluation von Nachhaltigkeit und dem Status Quo, der Zukunft und dem Innovationssystem der MEMS Industrie auseinandergesetzt, insbesondere hinsichtlich der Entwicklung des Marktes, der Technologie, der Förderlandschaft und etablierter Industrienetzwerke.

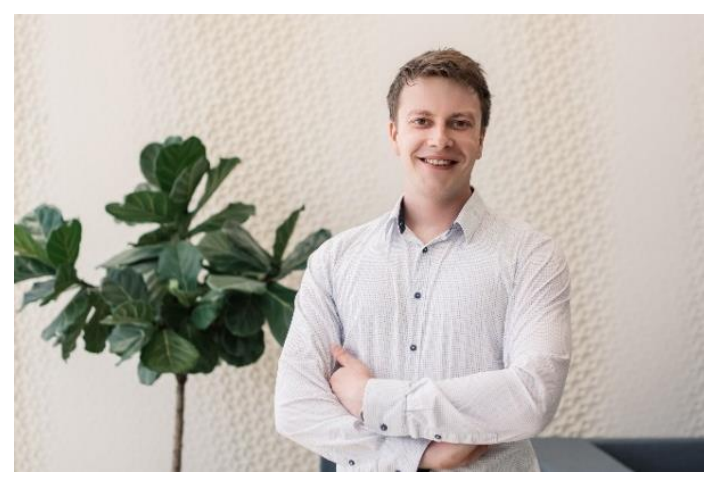

\section{Sc. David Kriebel}

Wissenschaftler |

Fachgebiet MEMS Simulation

David Kriebel war Nachwuchsforscher innerhalb des EPISA Projektes und war zuständig für den Entwurfsprozess der Inertialsensoren basierend auf der AlN Technologie, sowie für Druckwandler zum Einsatz in der Medizintechnik.

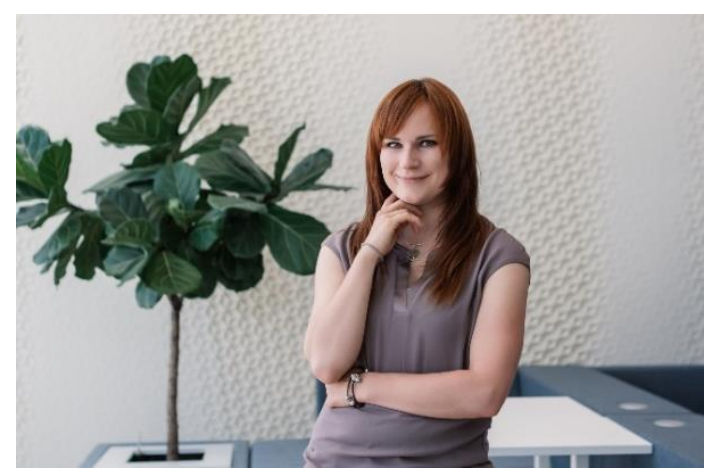

\section{Sc. Katja Meinel}

Wissenschaftlerin |

Fachgebiet piezoelektrische MOEMS

Katja Meinel war innerhalb des Projekts E-PISA als Nachwuchswissenschaftlerin tätig. Ihre Aufgabe im Projekt bestand in der Entwicklung eines MOEMSDemonstrators in Form eines Endoskop-tauglichen Gesamtsystems für die medizinische In-vivoDiagnostik. 

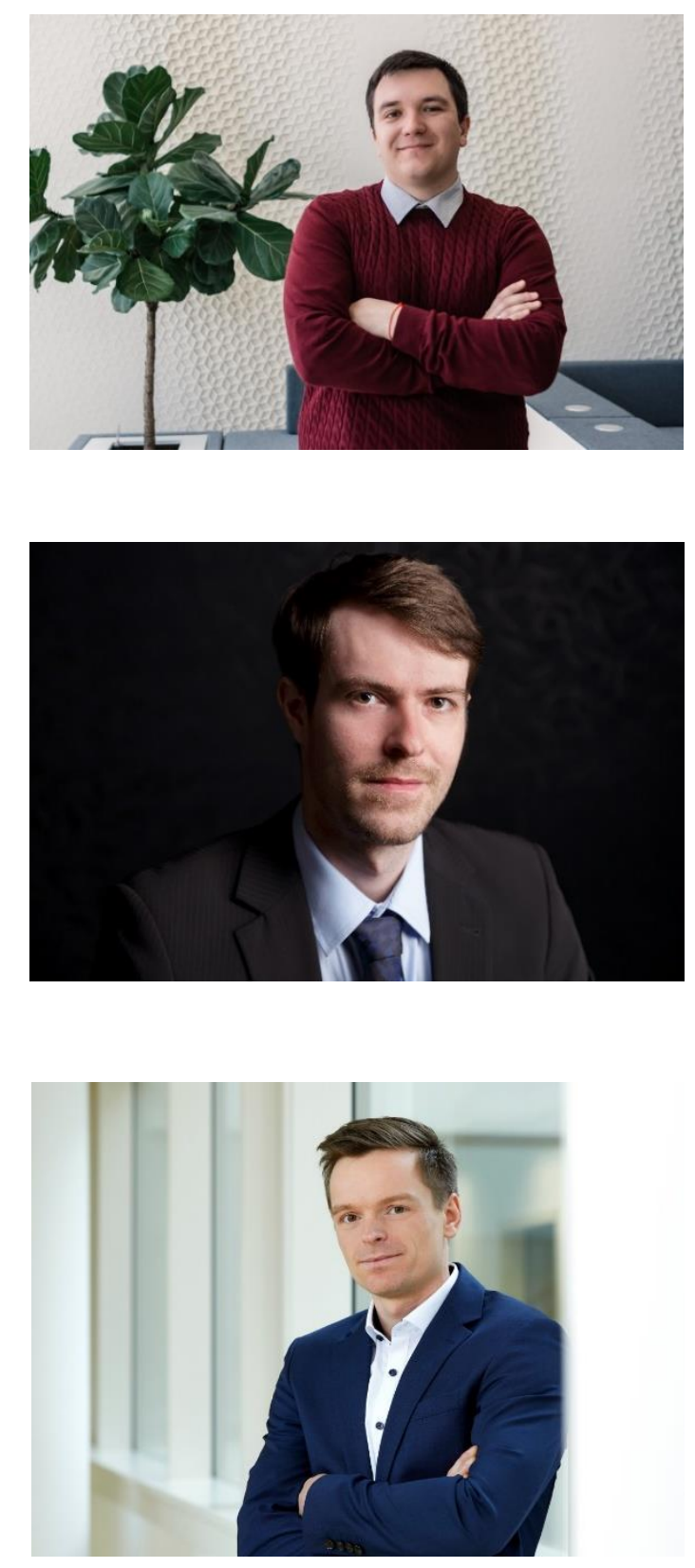

\section{Dr. Dmytro Solonenko}

Wissenschaftler |

Fachgebiet Grenzschichten und Dünnschichten

Dmytro Solonenko ist die wissenschaftliche Unterstützung für die Hauptrichtungen des Projekts: das Aufwachsen der hochwertigen AlN-Dünnfilme auf verschiedenen Substraten. $\mathrm{Zu}$ seinen Aufgaben gehört die Festlegung neuer Methoden und Verfahren zur Materialcharakterisierung für die reale Produktion.

\section{Sc. Martin Stiebing}

Wissenschaftler |

Fachgebiet Zuverlässigkeit von Mikrosystemen

Martin Stiebing untersuchte in der Nachwuchsforschergruppe vor allem die Zuverlässigkeit der realisierten Systeme und führte Ermüdungstests neuer Materialsysteme durch.

\section{Dr. Chris Stöckel}

Leiter Nachwuchsforschergruppe |

Fachgebiet piezoelektrische Mikrosysteme

Chris Stöckel war Projektleiter in der ESF geförderten Nachwuchsforschergruppe. Darüber hinaus hat Dr. Stöckel die AlN Technologie für die Projektpartner bereitgestellt und weiterentwickelt. Zusätzlich übernahm er die Forschung an potentiell energieautarken Wake-Up Mikrosystemen. 

Für Anwendungen in der medizinischen Diagnostik, IoT und Industrie 4.0 werden zukünftig smart systems benötigt, welche energieautark, drahtlos und stark miniaturisiert hergestellt werden können. 


\section{Innovative Applikationen}

Mikroelektromechanische Systeme (MEMS) sind bis zum heutigen Tag elementare Bestandteile in unserem Alltag geworden: Als Sensoren in Unterhaltungselektronik und der Medizintechnik sowie in der Industrie reicht ihr Einfluss von der Detektion von akustischen und mechanischen Schwingungen über die Erfassung von Bewegung, Temperatur und Feuchtemessung, über Gasanalytik bis hin zu mikrooptischen, medizintechnischen Produkten. Industrielle Roadmaps (Bosch, Intel, TI, HP, Yole, u.v.m.) prognostizieren ein exponentielles Wachstum auf bis zu einer Billion Sensoren pro Jahr bis 2021, getrieben von der Entwicklung des IoT (Internet-of-Things). Insbesondere für Anwendungen in der medizinischen Diagnostik werden zukünftig „,smart systems“ benötigt, welche energieautark, drahtlos und stark miniaturisiert hergestellt werden können. Beispiele hierfür sind kathetherbasierte Druckmessungen bzw. endoskopbasierte bildgebende Verfahren zur direkten (in-vivo) Anwendung im menschlichen Körper. Die Zielstellung der Arbeiten dieser Forschergruppe besteht darin, für diese Aufgabenstellungen Lösungsansätze zu erarbeiten und anhand von zwei Demonstratoren zu verifizieren, welche im Vergleich zum Stand der Technik eine stark miniaturisierte, nichtinvasive bzw. minimalinvasive Diagnostik und damit stark verringerte Belastung für den Patienten ermöglichen.
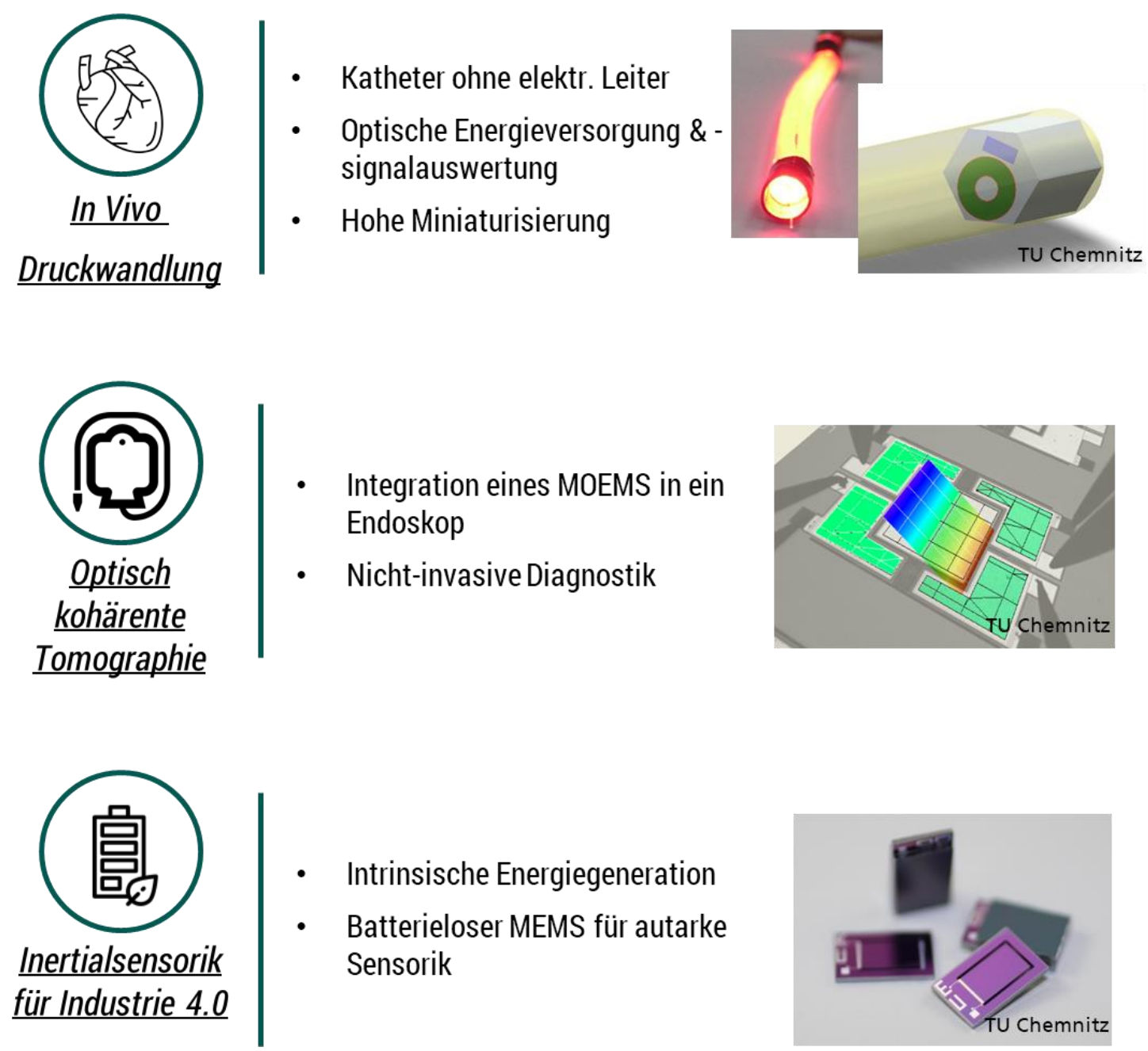


\section{Aluminiumnitrid-Dünnschichten für piezoelektrische Mikrosysteme kompakter Baugröße, hoher Leistungsdichte und intrinsischer Ladungsgeneration.}




\section{Innovative Technologien}

Allen Demonstratoren liegt als Lösungsansatz die Nutzung des piezoelektrischen Wandlerprinzips zugrunde. Damit können sowohl sensorische als auch aktuatorische Funktionalitäten, erzeugt werden. Durch das Anlegen einer elektrischen Spannung verformt sich das piezoelektrische Material und kann so als Aktor genutzt werden. Umgekehrt generieren piezoelektrische Kristalle bei Verformung eine Ladungsdifferenz, wodurch das Material als Sensor nutzbar wird. Im Vergleich zu den etablierten kapazitiven MEMS zeichnen sie sich durch eine kompaktere Baugröße und die Möglichkeit der intrinsischen Ladungsgeneration der sensorischen Komponente aus. Die Verwendung von AlN als piezoelektrisch aktives Wandlermaterial stellt im Gegensatz zur Verwendung von Materialien wie Blei-ZirkonatTitanat (PZT) und Polyvinylfluorid (PVDF) eine CMOS-kompatible, umweltfreundliche Alternative dar. Dadurch, dass AlN keine Curie-Temperatur besitzt ist es (hoch)temperaturstabil. Eine zusätzliche Steigerung der Empfindlichkeit der sensorischen Komponente entsteht durch die Kombination (monolithische Integration) von carbon nanotube (CNT)-basierten Transistoren als Verstärkerelemente in unmittelbarer Nähe (sehr kurze Signalwege) der elektromechanischen Wandlerelemente. Abb. 2 fasst das Innovationspotential des vorgesehenen Lösungsansatzes zusammen.

Stand der Technik

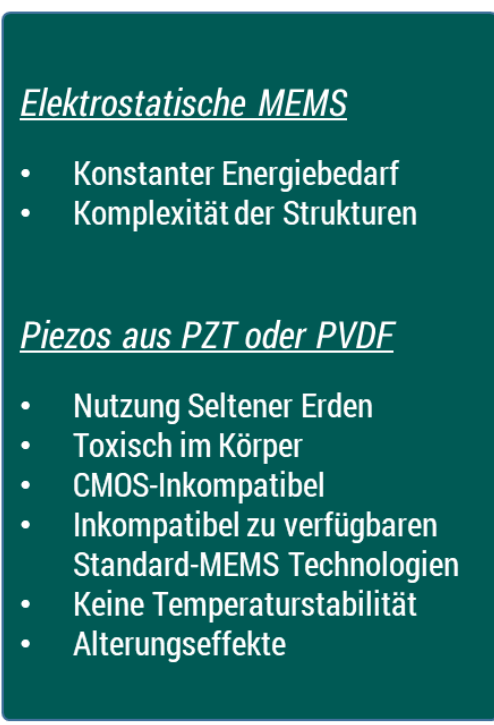

Märkte, Firmen und Bedarf

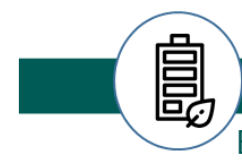

Energieeffizient Umweltfreundlich

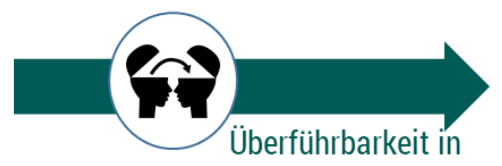
die Industrie

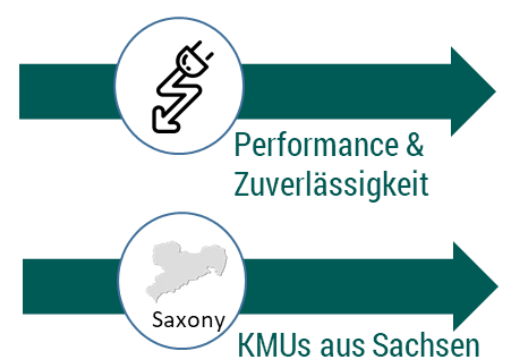

Innovationen

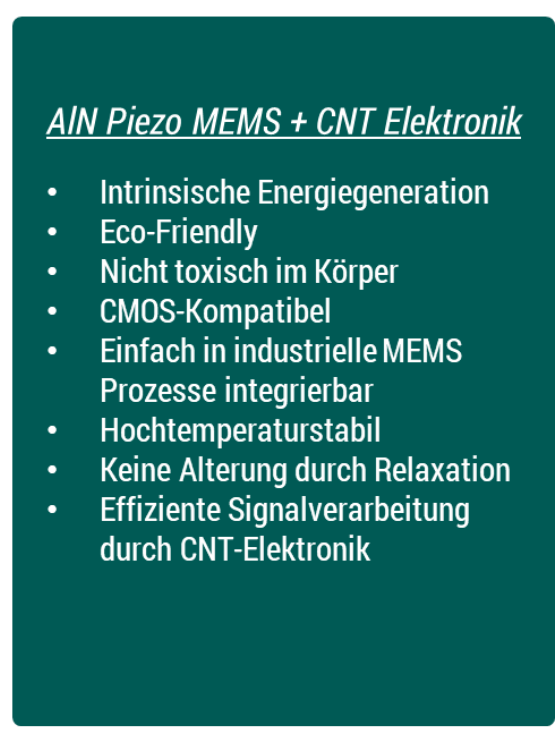


Die Forschungs- und Unternehmenslandschaft in Sachsen und insbesondere am Industriestandort Chemnitz bietet für die Nachwuchsforschergruppe ausgezeichnete Bedingungen, um innovative Sensorsysteme direkt in die Praxis zu transferieren. 


\section{Regionaler Bezug}

Das Forschungsvorhaben und die darin angestrebten technischen Entwicklungen sind auf dem Gebiet neuartiger MEMS Sensoren und Aktoren angesiedelt. Dieses Forschungsthema adressiert damit die Forschungsschwerpunkte „Materialien und Intelligente Systeme“ sowie „Mensch und Technik“ der TU Chemnitz und nutzt die hier vorhandenen Kernkompetenzen, um anwendungsnahe technologische Lösungen zu erarbeiten. Im Freistaat Sachsen gibt es auf diesem Gebiet diverse Schnittstellen mit Forschungseinrichtungen sowie mit traditionellen Industriebereichen, z.B. Automobilbau, Maschinenbau, Mikroelektronik und Textilindustrie, aber auch mit der stark wachsenden Medizintechnikbranche. Die Forschungs- und Unternehmenslandschaft in Sachsen und insbesondere am Industriestandort Chemnitz bietet für die Nachwuchsforschergruppe ausgezeichnete Bedingungen, um innovative Sensorsysteme direkt in die Praxis zu transferieren. Dadurch ergeben sich Verbesserungen in der Arbeitseffizienz, die zur Erhöhung der Wettbewerbsfähigkeit von Hochschule und Unternehmen führen.

Im Projekt und im Anschluss an das Projekt wurde speziell die Zusammenarbeit mit den sächsischen Firmen EDC, GEMAC und Fibragg (Elektronik und Sensorik für medizinische und industrielle Anwendungen), FHR (Anlagen- und Prozessentwicklung zu AlN) und dem Fraunhofer ENAS angestrebt. Darüber hinaus gibt es überregionale Kooperationen und Netzwerke mit Firmen wie Preciseley Microtechnology Inc. (Kanada), Biotronik (Deutschland), Panasonic (Japan) und vielen mehr.

Die Entwicklung mit Fokus auf medizintechnische Produkte und die Industrie 4.0 wird dazu beitragen, vorhandene Marktnischen frühzeitig zu besetzen und mit neuen Akzenten in der Forschung „Silicon Saxony“ als starkes europäisches Zentrum der Mikrotechnologie zu festigen. Darüber hinaus wurden die Themenfelder im Bereich Gesundheit und Demografie mit dem Schwerpunkt „Gesundes Leben“ in der High-Technik-Strategie der Bundesregierung als eine wichtige Zukunftsaufgabe formuliert.

Des Weiteren diente das Projekt im Besonderen der Qualifikation der Projektmitarbeiter auf exzellentem Niveau, so dass nach erfolgtem Abschluss ein Transfer von Personal und Ergebnissen in die lokale Wirtschaft (vgl. Operationelles Programm (OP) des Freistaates Sachsen für den ESF) aktiv gefördert wird. Die WissenschaftlerInnen haben durch eine Vielzahl von Veröffentlichungen sowie der Aufnahme, Durchführung und/oder dem Abschluss einer Promotion/Habilitation ihre akademische Laufbahn nachhaltig weiterentwickeln können. 


\title{
Die Nachwuchsforschergruppe hat lokal,
}

\author{
regional, national und global akademisches
}

Wissen transferiert und Impulse für industrielle

\section{Wertschöpfung für KMUs sowie für globale}

\section{Marktführer gesetzt.}

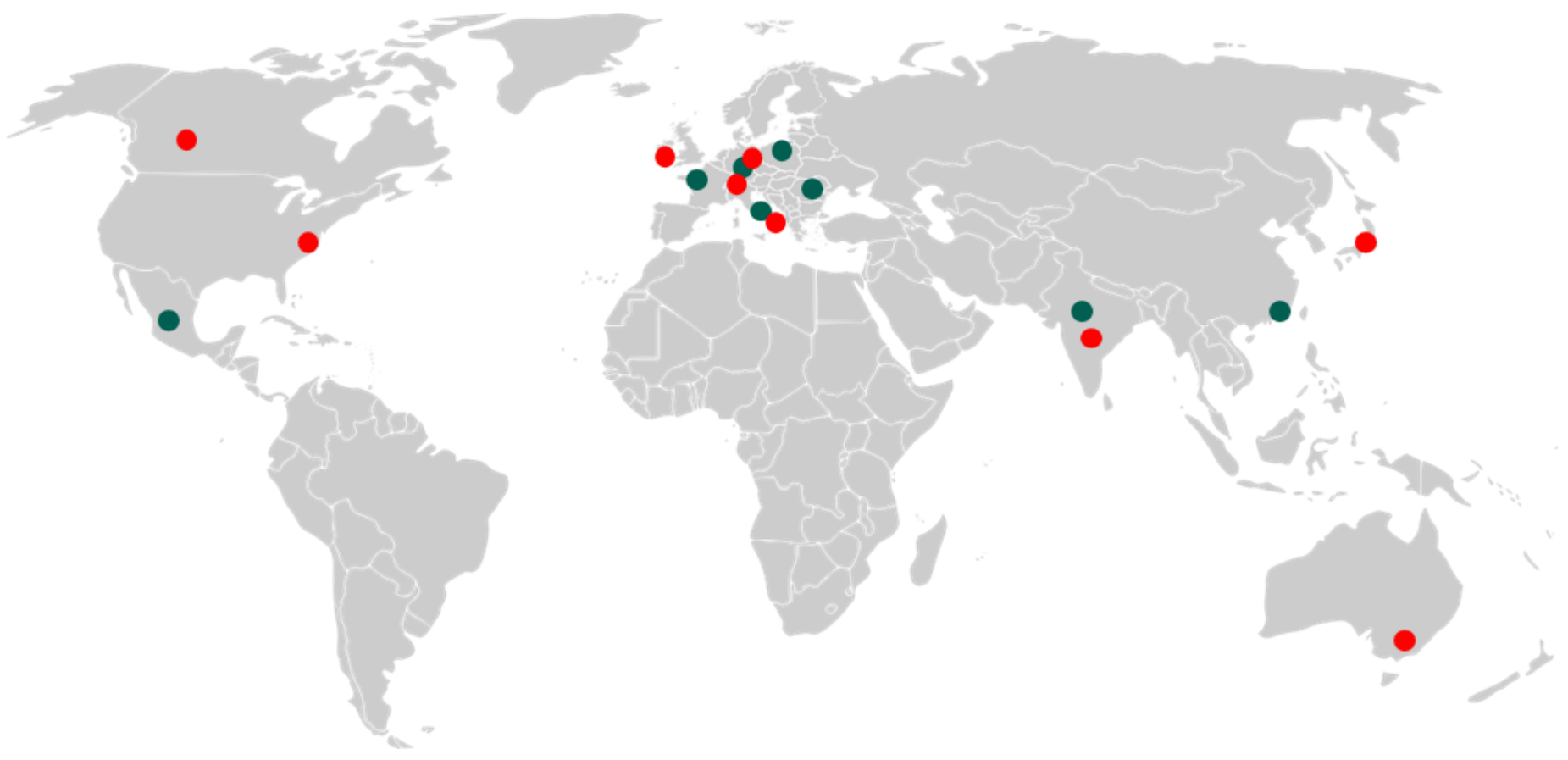

Auswahl an wissenschaftlichen Konferenzen:

IEEE Sensors 2018 | IEEE Sensors 2019 | DTIP 2018 | Transducer 2019 | EASS 2020 | R\&D Management 2019 | New Economic Sociology 2019 | CAS 2018 | DTIP 2019 MST Kongress 2019 | Flatlands Beyond Graphene | OSI 2019 | ICFSI 2019 | SSI 2018 | EASS Karlsruhe 2020

Auswahl an neuen Kooperationen / Netzwerken / Industrieanfragen:

Panasonic | Biotronik | Ruhruni Bochum | Preciseley Microtechnology | Electronic Design Chemnitz | TU Dresden | Picoquant | Fraunhofer | Stelect | ElbeValley | Sensry | NXP Semiconductors | X-FAB | Bosch Sensortec | ETH Zürich | Sindelhauser materials | Vathauer | Schaeffler | MicroSensys | Tagtron | Ulrich Rotte Anlagenbau | Dehn | PI Ceramics | Tata Steel 


\section{Wissenstransfer in Zahlen}

Die wissenschaftlichen Ergebnisse im Projekt E-PISA wurden vielfältig verwertet und das erworbene Wissen regional, europäisch und global transferiert.

Die Nachwuchswissenschaftler waren Bereich der akademischen Lehre und Weiterbildung des studentischen Nachwuchses aktiv. Es wurden eine Vielzahl von Übungen, Praktika und zwei neu entstandene Vorlesung gehalten. Zudem wurde rund ein Dutzend studentische Arbeiten betreut.

Darüber hinaus haben sich die Nachwuchswissenschaftler im Bereich der Lehre, des Projektmanagements und in den sozialen Kompetenzen weiterentwickelt und die Arbeit an insgesamt 5 Promotionen und 2 Habilitationen begonnen. Zwei Promotionen konnten in der Laufzeit von E-PISA abgeschlossen werden.

Internationale Sichtbarkeit haben die Forschungs- und Entwicklungsarbeiten des Teams vor allem durch Publikationen und Beiträge an wissenschaftlichen Konferenzen erhalten. Neben einen Best Poster Award und einer Einladung zur Veröffentlichung einer „Special Issue“Ausgabe bei Peer-Review Publikationen sind auch global wissenschaftliche Netzwerke geknüpft worden.

Besondere Aufmerksamkeit bekam die Forschungs- und Entwicklungsarbeit von regionalen, nationalen und globalen KMUs, sowie Global Playern. Hierbei hat die Kooperation mit dem Fraunhofer-Netzwerken geholfen die Sichtbarkeit der piezoelektrischen Mikrotechnologien zu erhöhen und in die industrielle Anwendung zu überführen. Die Grundlagen des Projekts E-PISA haben hierbei den Weg für Kooperationen mi Global Playern, wie Panasonic, geebnet. Darüber hinaus haben die Erkenntnisse, beispielweise zu Wake-Up MEMS, zu industrienahen Weiterführungsprojekten geführt, wie beispielweise die FORMIKRO Förderung des Bundes mit mehr als 10 deutschen Industriepartnern im Beitrat. Die Projektaktivitäten im Bereich der MOEMS Entwicklung haben zu Projektanbahnungen mit sächsischen, nationalen und internationalen KMUs, sowie neuen Netzwerken mit akademischen Partnern geführt.

Die medizintechnischen Entwicklungen mit Drucksensoren für Katheter ohne elektrische Kabel soll in eine EXIST-Ausgründung überführt werden.

Für eine weitere Anwendung von piezoelektrischen Mikrostrukturen ist eine Kooperation mit dem Fraunhofer ENAS und der Firma Biotronik für medizintechnische Produkte geplant.

Darüber hinaus sind von akademischen Partnern, Fraunhofer Instituten und von mehr als ein Dutzend Industriepartnern Anfragen zur Technologie, Kooperation oder Durchführung von F\&E Aufträgen bei den Wissenschaftlern der Nachwuchsforschergruppe eingegangen. Die im Projekt entwickelten Technologien und Applikationen sollen nachhaltig in die Anwendungen überführt werden um einen Mehrwert für Wissenschaft, Industrie und Gesellschaft zu erbringen. 


\section{Piezoelektrisches Aluminiumnitrid ermöglicht} neue, innovative Sensoren und Aktoren in der Mikrotechnik.

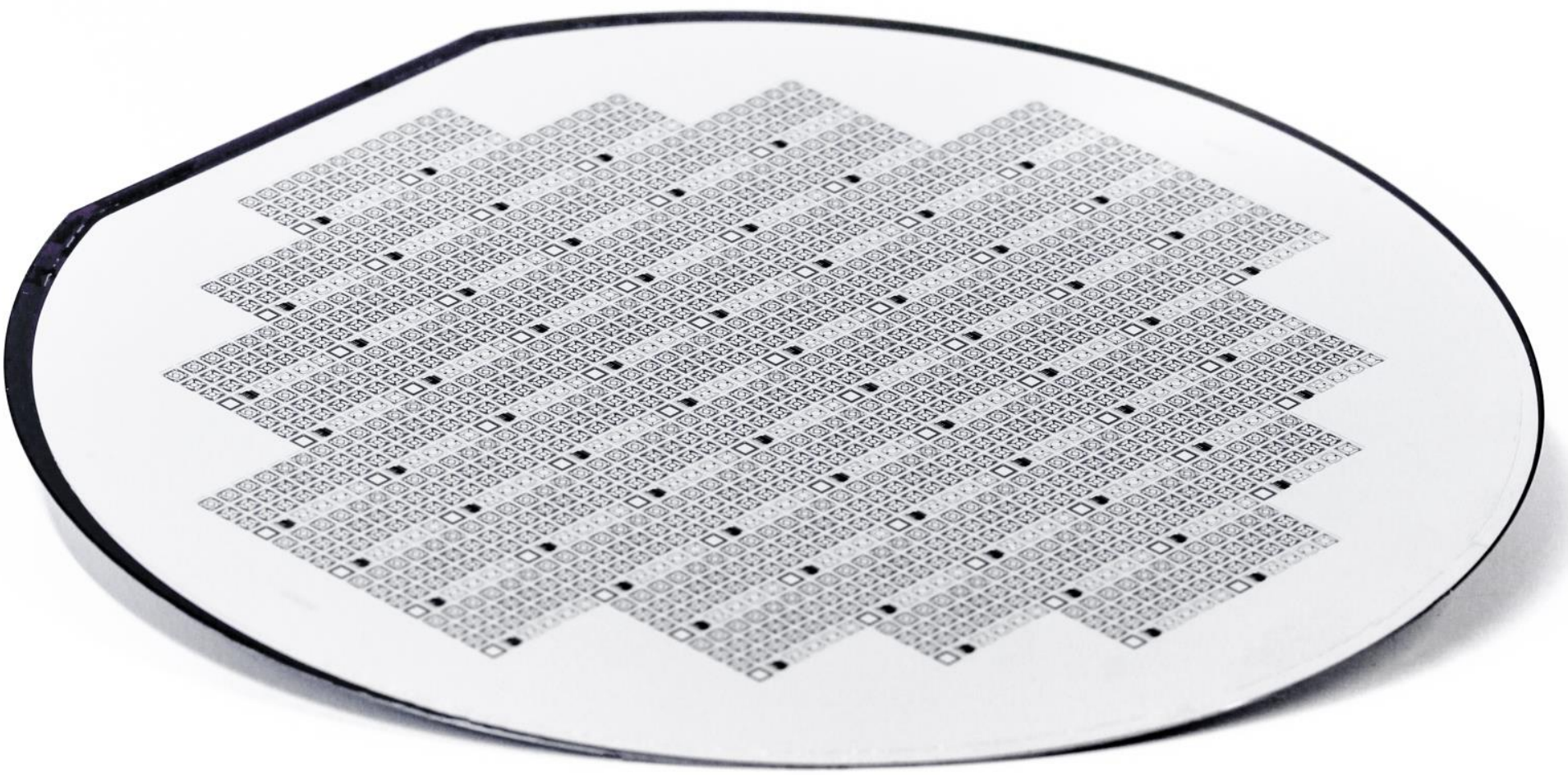




\section{Piezoelektrisches Dünnschicht-Aluminium- nitrid für Mikrosysteme}

Piezoelektrisches Dünnschicht-Aluminiumnitrid kann als Wandlermaterial für mikroelektromechanische Systeme (MEMS) genutzt werden. Neben den geringen Betriebsspannungen bietet der Wandler Vorteile durch eine Out-of-plane Aktuatorik und Sensorik. Gegenüber PZT basierte Materialien hat AIN keine Curie-Temperatur und ist temperaturstabil, zeigt keinen Versatz der Kristalle bei mechanischer Belastung oder Hysteresen und ist damit alterungsstabil. Da keine seltenen Erden oder giftigen Grundsubstanzen zur Abscheidung des III-V Halbleiters benötigt werden lässt sich das Material einfach in vorhandene Abläufe der Mikrotechnologie integrieren.

Im Rahmen der ESF geförderten Nachwuchsforschergruppe E-PISA wurden eine Reihe unterschiedlicher AlN basierter Technologien etabliert. Zunächst kann zwischen einer Technologie auf Basis von reinen Siliziumwafern und SOI Wafern (Silicon On Insulator) unterschieden werden. Die Abscheidung von AlN auf reinen Siliziumwafern erlaubt die Herstellung von SAW / BAW Strukturen und piezoelektrischen Ultraschallwandlern, also MEMS mit reinen Dünnschichten für hochfrequente und kleinste Applikationen. Zusätzlich wird über eine SOI-Wafer Technologie die Herstellung von MEMS ermöglicht, die ein definiertes Substrat erhalten. Dadurch können komplexe Federstrukturen, Massen und damit verbunden definierte Steifigkeiten, Bewegungsmodi und Resonanzen eingestellt werden, wie beispielsweise MOEMS, Wake-Up MEMS und Acoustic Emission MEMS.

Um AlN auf einen MEMS zu integrieren muss das Material unter Anderem zwischen zwei Elektroden abgeschieden und strukturiert werden. Wird ein elektrisches Feld zwischen den beiden Elektroden angelegt dehnt sich das Material und kann als Aktor genutzt werden. Wird das System gedehnt, generiert das piezoelektrische Material eine Ladung und die Dehnung kann dadurch sensorisch gemessen werden.

Eine im Projekt untersuchte Weiterentwicklung ist die Dotierung des AlN mit Scandium. Das Scandium verspannt das Kristallgitter, wodurch sich die Ladungsschwerpunkte stärker verlagern und höhere piezoelektrische Koeffizienten möglich sind. Das dadurch entstehende $\mathrm{AlScN}$ hat eine bis zu fünffach höhere Leistungsfähigkeit im Vergleich zu AlN. Im Projekt E-PISA wurde sowohl über Co-Sputtern, als auch über ein AlSc-Einzeltarget eine Abscheidung evaluiert. 
Die Verfügbarkeit einer hochwertigen AIN/AIScN Technologie wird von Industriekunden als kritische Voraussetzung für Mikroaktorik- und Sensorikanwendungen gesehen. 
Die größte Herausforderung ist es das AlN/AlScN in hoher Qualität in seiner CAchsenorientierung abzuscheiden, sodass das Material hohe piezoelektrische Koeffizienten aufweist. Die untere Elektrode fungiert hierbei nicht nur als elektrischer Kontakt, sondern auch als Wachstumsschicht. Im Rahmen des Projekts wurden zwei Wachstumsschichten für ein sehr gutes AlN Wachstum identifiziert. Zum einen konnte (111) orientiertes Pt optimiert werden. Die piezoelektrischen Koeffizienten sind homogen über den Wafer nahe des physikalischen Maximums. Darüber hinaus wurde eine Technologie mit (111)-Silizium als Wachstumsschicht erarbeitet. Die piezoelektrischen Koeffizienten sind hoch. Da das AlN direkt auf das Substrat aufgebraucht wird gibt es weniger Prozessschritte, was die ist die Technologie besser in industrielle MEMS Prozesse integrierbar und günstiger macht, sowie kürzere Prozessierungszeiten ermöglicht. Durch die Verringerung der Prozessschritte steigt auch die Ausbeute.

Die Technologie mit piezoelektrischen Dünnschicht-AlN ist zusätzlich robust gegenüber Hochspannung ausgelegt worden. In Dünnschichtsystemen bilden sich bei hohen elektrischen Spannungen elektrische Pfade im Material aus, die zu einer Reduktion der Performance und schlussendlich zu einem Ausfall führen. Dieses Phänomen konnte durch eine Optimierung des AlN und durch eine Etablierung einer Schutzschicht gegenüber Kurzschlüssen reduziert werden. Es gibt ausschließlich $2 \%$ Ausfälle piezoelektrischer Strukturen bei elektrischen Spannungen von bis zu 20 Volt. 90 \% Der Strukturen können bei bis zu 50 V kurzschlussfest arbeiten. Einige Chips konnten bis $200 \mathrm{~V}$ betrieben werden. (Daten prüfen und neue Daten einfügen)

Eine weitere Innovation bei der Integration von piezoelektrischem Dünnschicht AlN und den Elektroden ist die Weiterentwicklung der Strukturierungstechnologien. Eine Einführung der trockenchemischen Strukturierung von AIN und Platin-Elektrode führt zu einer Reduktion der Ätztoleranzen um einen Faktor von 10 bis 50. Die Ätztoleranz konnte auf unter $200 \mathrm{~nm}$ gesenkt werden. Dadurch ist es möglich in Zukunft kleinere, präzisere und zuverlässige Aktoren, Sensoren und Leitbahnen zu designen und auch auf schmalsten Federstrukturen zu integrieren, sowie Nanostrukturen herzustellen.

Die technologischen Innovationen konnten noch im Projekt direkt in Mikrosysteme integriert werden. Dadurch sind neue, innovative mikro-opto-elektro-mechanische Systeme (MOEMS), Hochfrequenz-MEMS (SAW MEMS), Kleinste Membranwandler für Kathetersensorik und Wake-Up Mikrosysteme entstanden. 
Erstmals konnten piezoelektrische 2D MOEMS mit Scanwinkeln über $120^{\circ}$, einem $4 \mathrm{~mm}^{2}$ Footprint und einer integrierten Sensorik aufgezeigt werden. 


\section{Mikro-opto-elektro-mechanische Systeme}

Der Einsatz von Mikrospiegeln ist Voraussetzung zahlreicher photonischer Anwendungen, in denen sie als ein- oder zweidimensionale Ablenkeinheit für die statische Justierung oder das dynamische Ablenken (Scannen) eines Laserstrahls fungieren. $\mathrm{Zu}$ den potentiellen Anwendungen gehören optische Verfahren der Biophotonik, wie bspw. die optische Kohärenztomografie und Fluoreszenzmikroskopie zur Aufnahme höchstauflösender 2D- und 3D- Gewebsabbildungen zur Diagnose onkologischer und dermatologischer Erkrankungen. Das Anwendungsspektrum von Mikrospiegeln umfasst ebenso die Bereiche der Messtechnik zur 2D- und 3D-Profilmessung unterschiedlichster Objektoberflächen sowie die Analytik für den Einsatz in spektroskopischen Verfahren. Im Konsumentenbereich werden die Mikrosysteme als Lichtablenkeinheit in Projektionsdisplays und Laserscannern sowie für das autonome Fahren (LIDAR) eingesetzt.

Aus den vielfältigen industriellen Anwendungen ergeben sich die Anforderungen einer unkomplizierten Herstellung, einer RoHS-kompatiblen Technologie, Energieeffizienz sowie ausgezeichneter Leistungsparameter, Dynamik und Präzision an derartige Mikro-Optiken. Durch piezoelektrische Aktoren auf Basis von Aluminiumnitrid können die geforderten Spezifikationen aufgrund der exzellenten Eigenschaften des Wandlermaterials vereint werden. Die monolithische Integration von Positionssensoren bietet darüber hinaus die Möglichkeit eines Closed-Loop-Betriebs zur Erzielung hoher Präzision für Strahljustierung und Bildgebung.

Auf Basis der Vorgaben der Humanmedizin an endoskoptaugliche Scannersysteme wurden die resonanten Scanner mit vier AlN-Aktoren und einem Spiegeldurchmesser von $700 \mu \mathrm{m}$ in einer Gesamtchipgröße von $2 \mathrm{~mm}$ x $2 \mathrm{~mm}$ umgesetzt. Es existieren Spiegelvarianten unterschiedlicher Federbreite und variierenden exzentrischen Massekörpern zur Realisierung verschiedener Scanpattern und Arbeitsfrequenzen. Die fabrizierten Mikrospiegel zeigen mit einem Scanwinkel über $120^{\circ}$ bei $50 \mathrm{~V}$ und $13 \mathrm{kHz}$, der Fähigkeit in zwei Dimensionen zu scannen sowie der Möglichkeit des Einsatzes im Closed-Loop-Betrieb, eine exzellente Performance im Vergleich zum Stand der Technik.

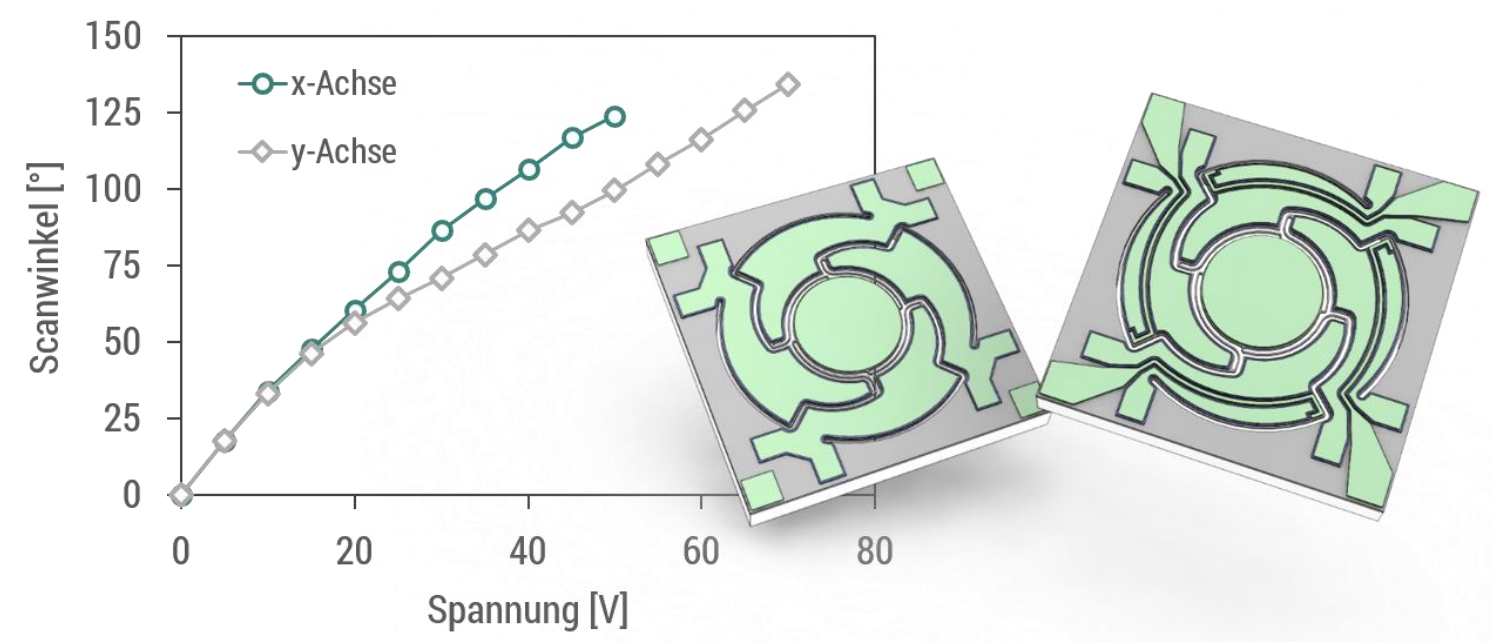




\section{Die MOEMS Technologie erlaubt eine}

Miniaturisierung der Optik zur Integration in

Endoskope mit einem Durchmesser unter 5 mm. 
Durch die Integration in endoskopische Sonden kann das diagnostische Potential medizinischer Bildgebungsverfahren, wie der OCT und der Fluoreszenzmikroskopie ausgeschöpft und deren Anwendungsbereich stark erweitert werden, da somit die Diagnostik mittels Echtzeitvisualisierung auch innerhalb des Körpers erfolgen kann. Für die Umsetzung miniaturisierter Sonden, ist der Einsatz von Mikrospiegeln als Lichtablenkeinheit zum Abrastern des Gewebes und gleichzeitig zum Einsammeln des dabei zurück reflektierten bzw. emittierten Lichts nötig.

Zur Evaluierung der Funktion des in E-PISA entwickelten Mikrospiegels als 2D-Scaneinheit für die Medizintechnik wurde unter Berücksichtigung medizintechnischer Anforderungen ein Funktionsdemonstrator in Form einer Sonde für die endoskopische Fluoreszenzmikroskopie, die hier beispielhaft für scannende, optische Verfahren der Medizintechnik vertreten ist, entwickelt.

Der 2D-Scannerspiegel wurde zur Umsetzung eines frontalabbildenden Aufbaus in einem Winkel von $45^{\circ}$ im Kopf der Sonde eingebaut. Eine GRIN-Optik mit Prisma zur Strahlumlenkung fokussiert das Laserlicht auf die Spiegeloberfläche, die das Licht auf die Probe reflektiert. Das von der Probe emittierte Fluoreszenzlicht wird über denselben Pfad zurückgeführt, detektiert und verarbeitet. Eine softwareseitige Bildzuordnung des detektierten Signals erfolgt mithilfe des Feed-Back-Signals der integrierten Positionssensoren.

Der Entwurf des Endoskopkopfs wurde unter Einhaltung der Vorgaben der Humanmedizin so optimiert, dass eine möglichst große Numerische Apertur durch einen minimalen Arbeitsabstand erzielt wird. Der Außendurchmesser konnte dahingehend auf einen Wert von 4,8 mm angepasst werden. Mit einem berechneten Airy-Radius von 3,2 $\mu \mathrm{m}$ kann ein Bildfeld von mehr als 100 x 100 Pixeln bei einer Bildwiederholungsrate von $250 \mathrm{fps}$ erzeugt werden.

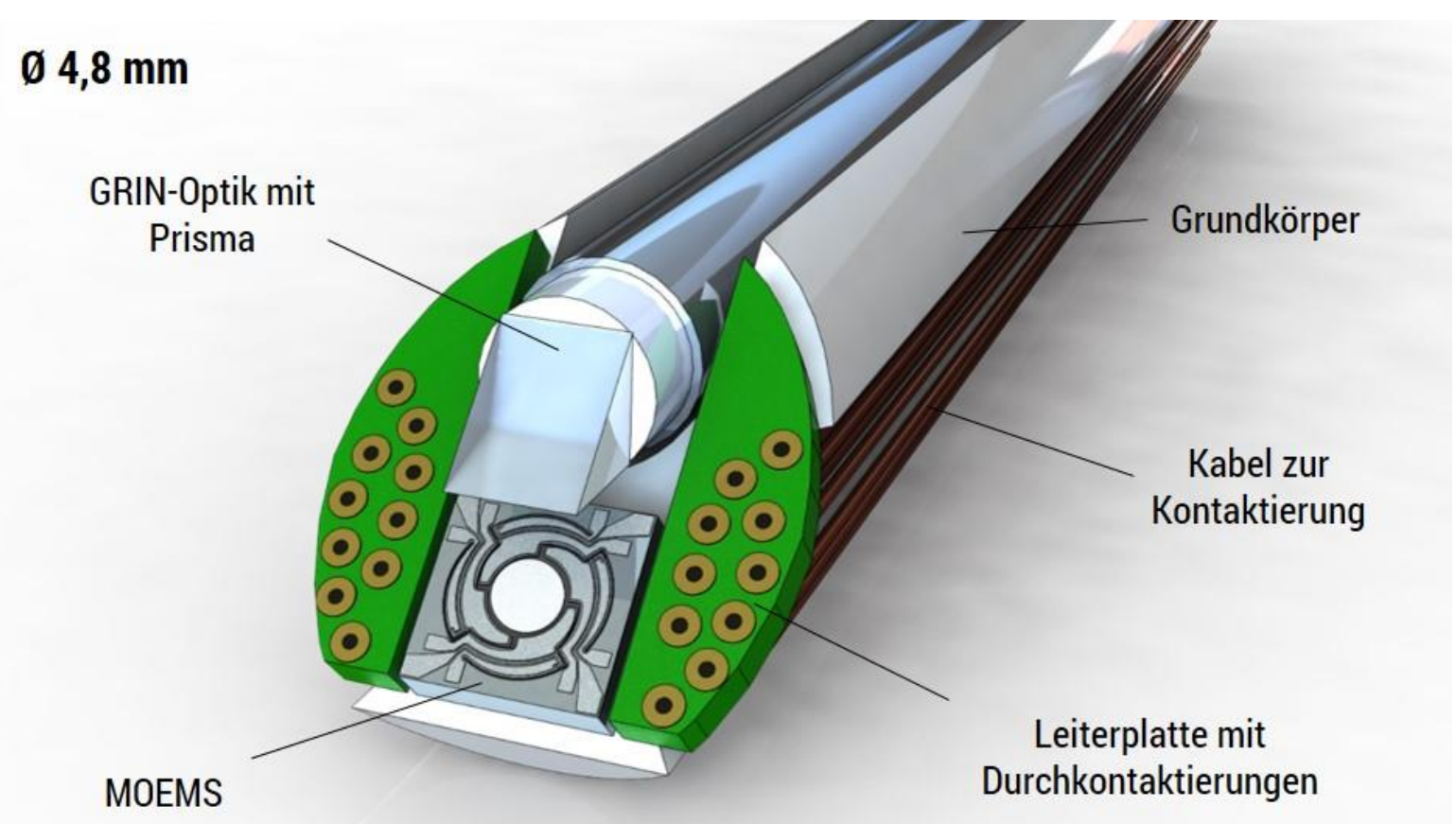






\section{Körperschallanalyse \\ für \\ eine \\ optimierte \\ Zustandsüberwachung neuer Sensoren der \\ Industrie 4.0}

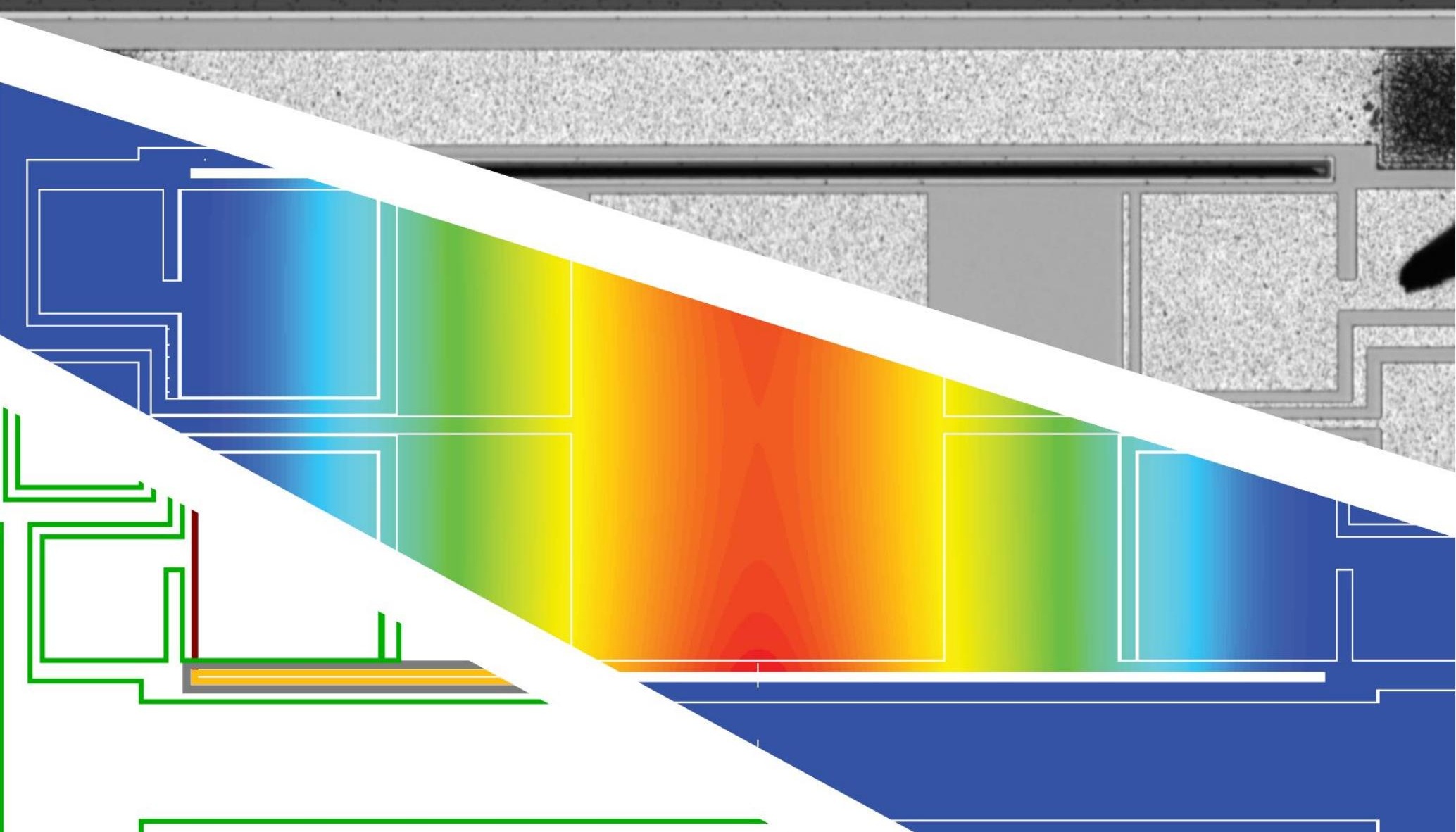




\section{Acoustic Emission Sensoren}

Die Zustandsüberwachung von Maschinen oder auch Gebäuden gewinnt zunehmend an Bedeutung. Dadurch können rechtzeitig Beschädigungen oder Verschleiß erkennen und prognostiziert werden. Die hier auftretenden hochfrequenten Körperschallwellen weisen in der Regel sehr kleine Amplituden sowie sehr kurze Zeitsignale auf. Diese permanente Messung kann durch hochempfindliche Beschleunigungssensoren realisiert werden. Eine Lösung bietet dabei die Verwendung mikroelektromechanischer Systeme (MEMS). Diese weisen bei hoher Stückzahl wesentlich geringere Herstellungskosten als kommerzielle Körperschallsensoren/Acoustic-Emission-Sensoren (kurz AE-Sensoren) auf.

Im Rahmen des Forschungsprojektes wurden verschiedene Varianten AE-Sensoren entworfen und untersucht. Die Resonanzfrequenz der AE-Sensoren wurde auf $100 \mathrm{kHz}$ festgelegt. In diesem Bereich befindet sich die Hauptkomponente des AE-Signals. Die Bandbreite sollte möglichst hoch sein und die Einschwingzeit möglichst gering, um zeitlich kurze AE-Signale zu erfassen. Eine geringe Einschwingzeit kann durch eine geringe Güte des mechanisch resonanten Systems eingestellt werden.

Die Sensorvarianten ergeben sich aus einem direkten und einem indirekten Auswerteprinzip. Technologisch bedingt können nur out-of-Plane Verformungen detektiert werden, was bei allen Designs berücksichtigt werden muss. Bei der direkten Auswertung befindet sich ein piezoelektrisches Sensorelement auf einer verformbaren Struktur. Diese Struktur wird bei Auftreten eines AE-Signals verformt und das Sensorelement generiert eine Ladung proportional zur Auslenkung. Es wurden 2 verschiedene Design dazu entworfen. Um die gesamte Fläche des beidseitig eingespannten Biegebalkens auszunutzen, wurde das Sensorelement in zwei Bereiche aufgeteilt. Damit können Verformungen, induziert durch Zug- und Druckspannungen, detektiert werden. Durch eine elektronische Reihenschaltung dieser Elemente können die elektrischen Ladungen aufaddiert werden und das Detektionssignal maximiert werden. Messungen ergaben eine ausreichende Übereinstimmung mit den festgelegten Spezifikationen. Aufgrund sehr geringer Verformungen konnte auch ein weitgehend lineares Verhalten über den relevanten Frequenzbereich nachgewiesen werden.
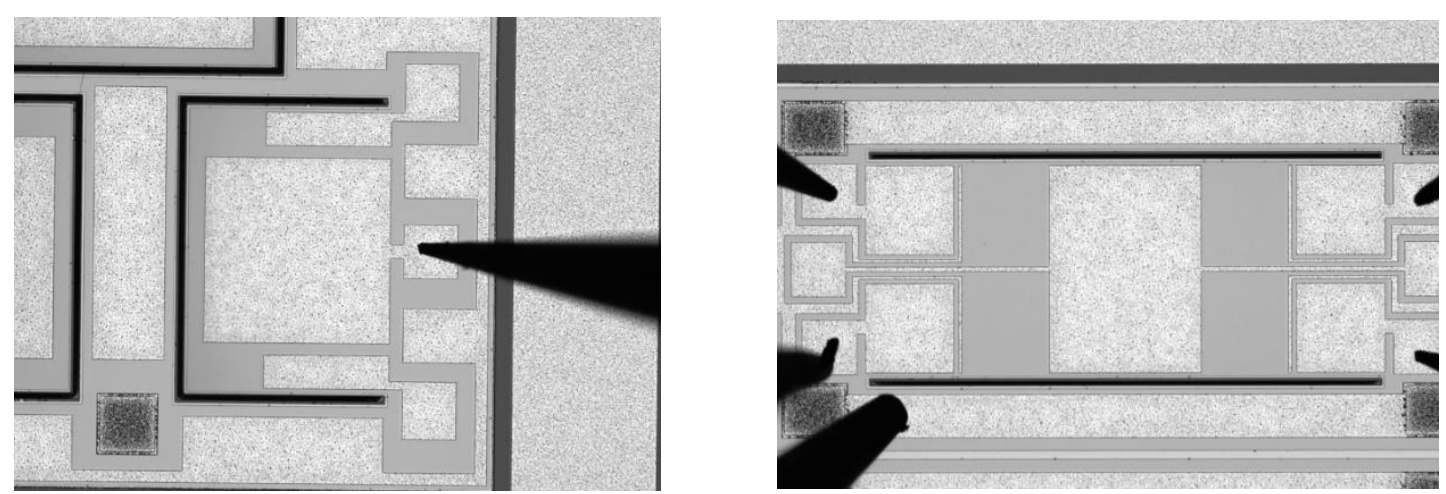

Variante 1 - einseitig eingespannt (links), Variante 2 - beidseitig eingespannt (rechts) 
Akustische Oberflächenwellen zur Messung von Körperschall ermöglichen eine weitere Verbesserung der Genauigkeit von Sensorsystemen. 
Beim indirekten Auswerteprinzip wird eine Oberflächenwelle (Surface-AcousticWave/SAW) mit Hilfe piezoelektrischer Aktuatoren generiert. Diese wird über eine mechanisch verformbare Struktur gesendet und am anderen Ende mit Hilfe einer baugleichen Aktuatorstruktur gemessen. Daher muss zum einen die verformbare Struktur aus einem beidseitig eingespannten Biegebalken bestehen und zum anderen müssen die SAWAktuatorstrukturen außerhalb dieses Bereiches liegen, um nicht selbst eine Verformung zu erfahren. Die aktiven piezoelektrischen Strukturen wurden als Fingerstrukturen, sogenannte Interdigitaltransducers (IDT), ausgeführt. Dabei bestimmt der Abstand der Fingerstrukturen untereinander die Wellenlänge der SAW. Aufgrund der genutzten Herstellungstechnologie wurde eine Wellenlänge von $40 \mu \mathrm{m}$ festgelegt (SAW Frequenz $125 \mathrm{MHz}$ ). Demnach bewegt sich die SAW mit $5000 \mathrm{~m} / \mathrm{s}$ über die verformbare Struktur und wird am anderen Ende des Balkens durch einen weiteren IDT erneut gemessen. Durch Verformung der Struktur kommt es schließlich zu einer Phasenverschiebung zwischen dem angelegten Eingangssignal und dem messbaren Ausgangssignal. Diese Phasenverschiebung ist proportional zur Auslenkung des Balkens. Dieses Verfahren sollte eine höhere Empfindlichkeit als das direkte Auswerteverfahren aufweisen.
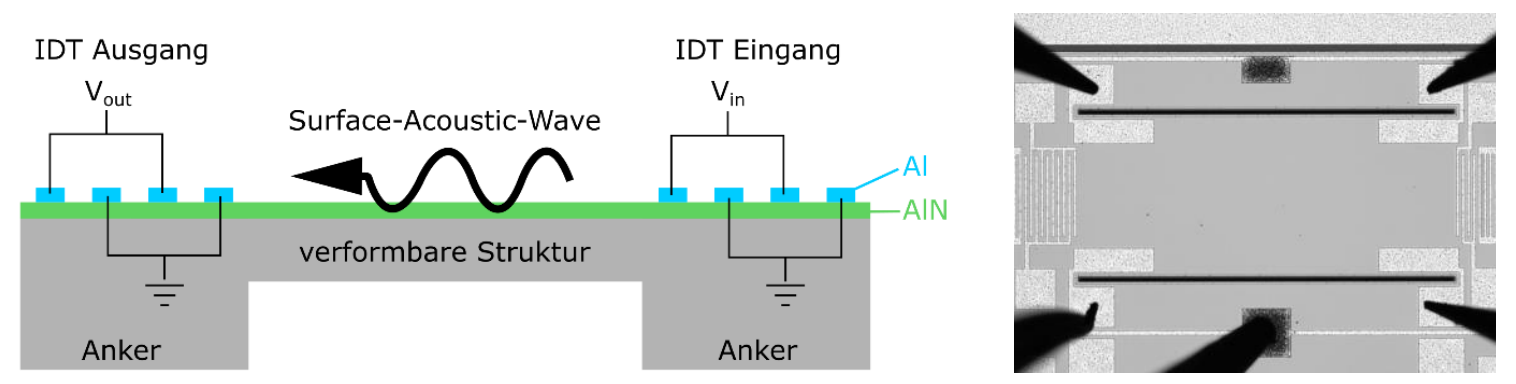

AE-SAW-Prinzip (links), AE-SAW-Sensor Messung (rechts)

Technologisch bedingt ergab sich keine Möglichkeit eine geforderte Güte von 10 bis $20 \mathrm{zu}$ realisieren. Aus diesem Grund wurde ein weiteres Konzept zur Dämpfungsgenerierung in piezoelektrischen MEMS umgesetzt und auf alle Sensorvarianten angewandt. Dieses Konzept wird als aktive Dämpfung bezeichnet. Dabei wird das eigentliche Sensor-Ausgangssignal dazu verwendet, eine geschwindigkeitsproportionale Spannung zu generieren, welche auf ein entsprechendes Aktuatorelement rückgekoppelt wird. Diese Umwandlung erfolgt mit Hilfe eines Reglerschaltung, bestehend aus einem Ladungsverstärker, Differentiator und Spannungsverstärker. Mit Hilfe eines mehrstufigen Spannungsverstärkers kann eine einstellbare Dämpfungskraft in einem closed-loop-System erzeugt werden.

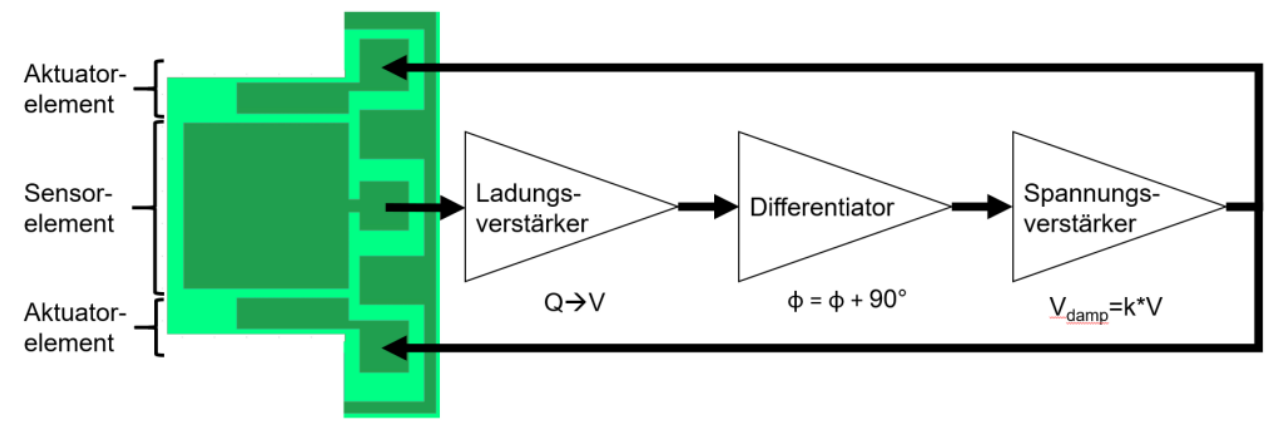

Prinzip der aktiven Dämpfung für piezoelektrische Beschleunigungssensoren 
Mikrosysteme mit optischer Lagedetektion zur Miniaturisierung von Katheterleitungen.
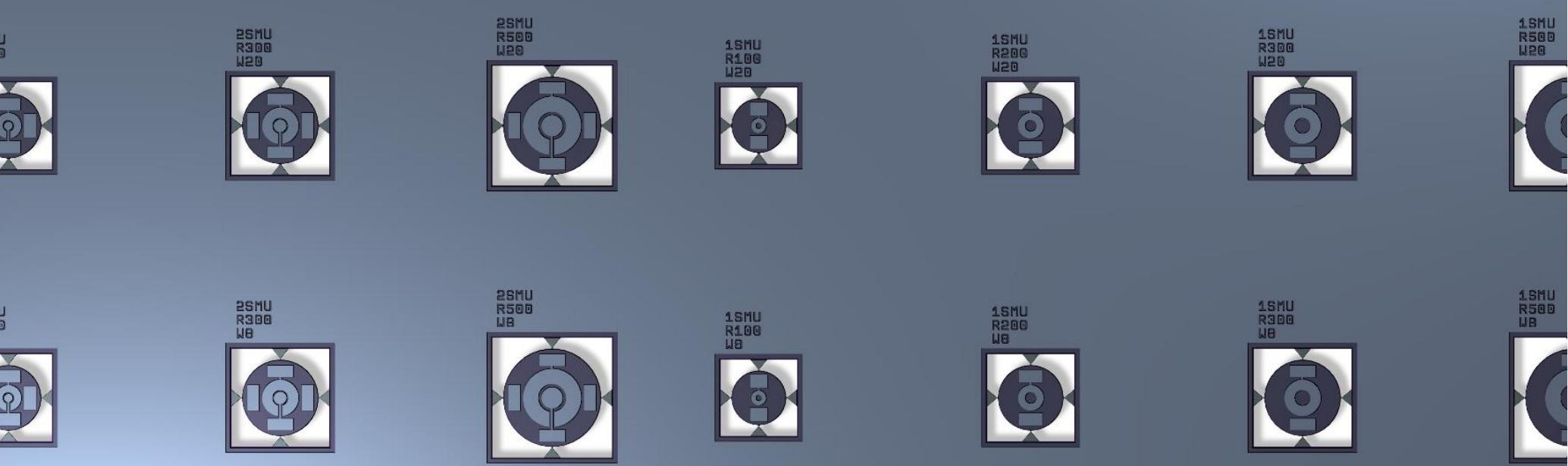

2)
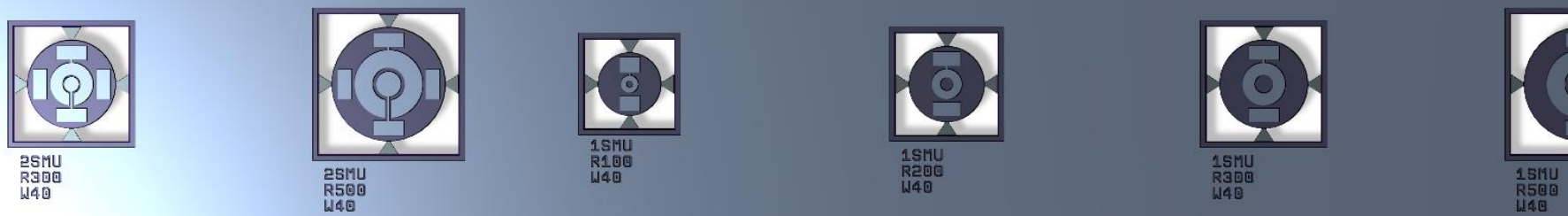


\section{Drucksensoren für medizinische Katheter}

Sensorik nimmt auch in der Medizintechnik an Bedeutung zu. Bei den immer fortgeschrittenen Eingriffen kommt es vor allem auf Bauform bzw. -größe sowie Flexibilität der eingesetzten Sensoren an. Ein Beispiel soll hier die katheterbasierte Druckmessung zur direkten (in-vivo) Anwendung im menschlichen Körper sein. Dabei soll ein Drucksensor in einen Herzkatheter integriert werden und zum Beispiel bei koronaren Herzkrankheiten zum Einsatz kommen könnte. Der Sensor wurde als Membranstruktur umgesetzt.

Diese Drucksensormembran wird direkt in den Katheterkopf eingelassen. Die Membran besitzt technogisch bedingt keine eigene Kavität, wodurch ein Absolutdruck gemessen werden kann. Diese Verformung wird optisch detektiert. Im Vergleich zu bisherigen Technologien entfallen für die Katheterleitung elektrische Anschlüsse, wobei ein erhebliches Platzersparnis gewährleistet werden kann. Die optische Lagedetektion erfolgt nach einer Art Fabry-Pérot-Interferometer. Die Membran selbst besteht aus einer dünnen SiO2-Schicht sowie der piezoelektrischen AlN-Schicht. Dadurch ist die Struktur hochsensibel selbst zu sehr kleinen Druckveränderungen. In Verbindung mit der verwendeten optischen Lagedetektion kann so ein hochauflösender Drucksensor realisiert werden.

Auf der Membran wurden piezoelektrische Aktuatoren integriert, welche zur Lageregelung genutzt werden. Die Energieversorgung dieser Aktuatorstrukturen erfolgt mit Hilfe einer optischen Energieversorgung.

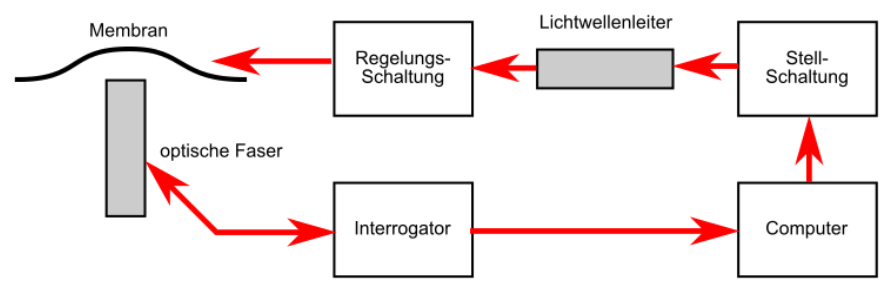

Messprinzip Drucksensor in Herzkatheter

Im Rahmen des Forschungsprojektes wurden verschiedene Varianten, sowie Membrangrößen gefertigt. Die kleinste gefertigte Membran weißt dabei einen Durchmesser von $100 \mu \mathrm{m}$ und einen Chipdurchmesser von $500 \mu \mathrm{m}$ auf. Diese und weitere Designs sind damit geeignet, in einem Herzkatheter zum Einsatz zu kommen, da dieser typischerweise einen Außendurchmesser von $1 \mathrm{~mm}$ aufweist. Es konnte experimentell Temperatur- sowie Umgebungsdruckeinflüsse auf die Membran bzw. Messstrecke nachgewiesen und kompensiert werden.
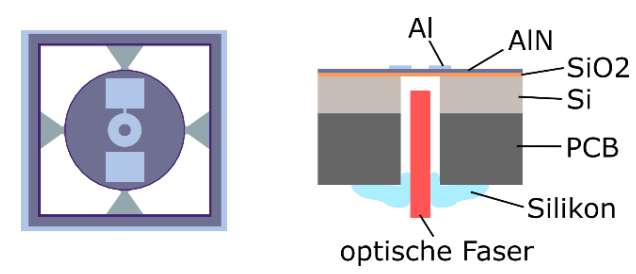

Membran Draufsicht (links), Messstrecke innerhalb der Katheterleitung (rechts) 
Piezoelektrische Wake-Up MEMS haben die Fähigkeit energieautonom inertiale Signale zu detektieren und somit batteriefreie Inertialsensorik zu ermöglichen. 


\section{Wake-Up MEMS}

Moderne, intelligente und autonome Systeme in der Mobilität, IoT und Industrie 4.0 integrieren sich in einer exponentiell wachsenden Stückzahl in unserer Arbeits- und Umwelt. Viele Systeme vernetzen sich drahtlos. Die Energieversorgung ist zumeist eine kritische Komponente. Lösungen für möglichst energieautonome Systeme sind gefordert.

Ein Ansatz Systeme energieeffizient und autonom zu gestalten ist das System in einen Dornröschenschlaf $\mathrm{zu}$ versetzen, wenn es nicht gebraucht wird und damit den Energiespeicher zu entlasten. Gleichzeitig muss das System permanent fähig sein seine Messaufgabe zu erfüllen. Dies kann zum Beispiel ein Schock oder eine kritische Vibration an einer Maschine, einem Achslager oder einer Infrastruktur sein. Tritt ein Ereignis, beispielsweise ein mechanischer Schock ein, weckt ein piezoelektrisches Mikrosystem, Wake-Up MEMS, eine Elektronik auf und diese kann das Ereignis speichern, senden und / oder mit weiteren Sensoren genauer messen.

Hierbei eignen sich piezoelektrische Mikrosysteme optimal. Die piezoelektrischen Wandler generieren bei mechanischem Stress oder Deformation eine elektrische Ladung / elektrische Spannung. Um in seiner Umgebung nach Schocks oder Vibrationen zu detektieren muss dem piezoelektrischen Mikrosystem keine elektrische Hilfsenergie zugeführt werden. Der MEMS ist energieautonom. Durch Integration der piezoelektrischen Wandler auf ein siliziumbasiertes Feder-Masse-System kann das MEMS auf vielfältige Schock- und Vibrationssignale abgestimmt werden.

Die bisher vorhandenen MEMS waren als eindimensionale Feder-Masse-Systeme mit einer Masse und einer Feder aufgebaut. Verschiedene Designs für eindimensionale Messung eines inertialen Signals wurden für verschiedene Anwendungen als Wake-Up MEMS ausgelegt und vermessen. Darüber hinaus ist ein Design für dreidimensionale Messung (eine Raumrichtung und zwei Drehrichtungen) einer Vibration oder eines Schocks entworfen und charakterisiert. Das System besteht aus einer zentralen Masse mit vier Sensorfedern. Je nach Amplitude und Phase der einzelnen Sensorfedern auf einen Schock oder eine Vibration kann eine von drei Raumrichtungen identifiziert werden.

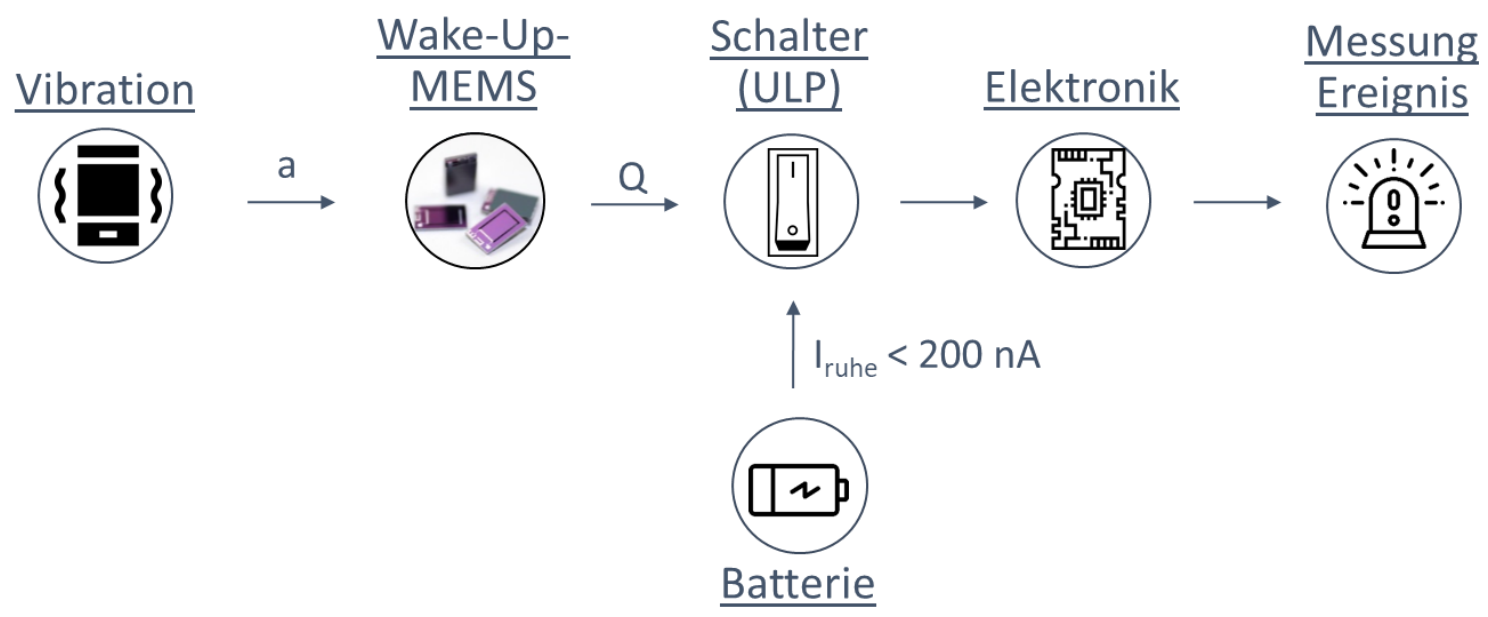




\section{Das Verständnis des Wachstums der Dünnschichten auf} Kristallebene ist ein Schlüssel für eine leistungsfähige Mikrotechnologie.

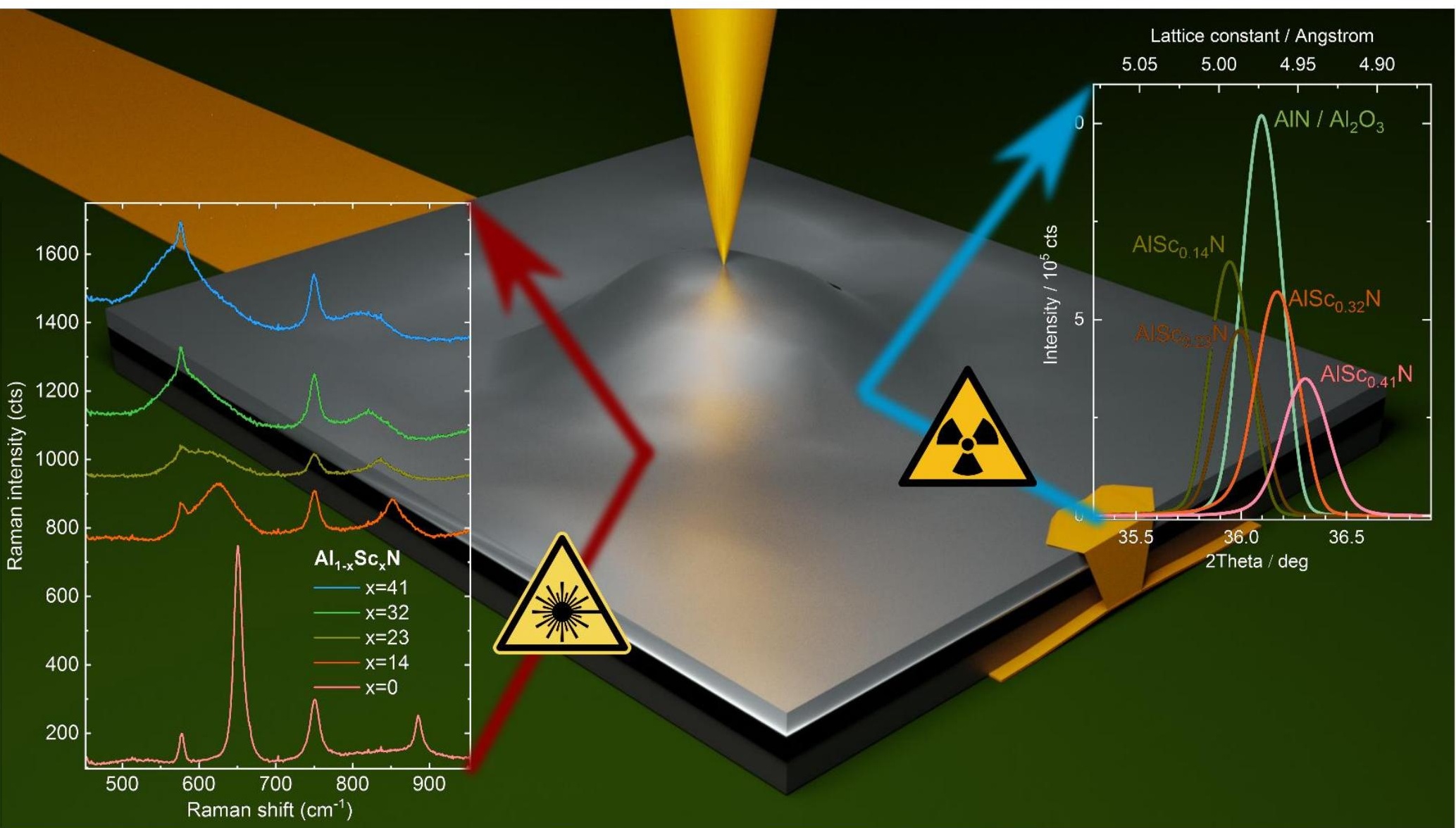




\section{Kristallwachstum}

Aluminiumnitridkristalle haben ein komplexes Kristallwachstum, das für zahlreiche Abscheide- und Umgebungsparameter empfindlich ist. Das Magnetron-Sputtern wird zum Abscheiden dünner kristalliner Schichten im Wafer-Maßstab aufgrund seiner industriellen Kompatibilität und Effizienz verwendet. Trotz der komplexen Wachstumsoptimierung wurden qualitativ hochwertige Proben von AlN-Dünnfilmen hergestellt. Um zu beurteilen, ob die Beschichtung dem Zweck der aktiven Schicht in MEMS dient, wurden mit dem EPISA-Projekt schnelle Analysemethoden zu ihrer Charakterisierung entwickelt. Die Identifikation von piezoelektrischen Koeffizienten wurde bisher über miniaturisierte, mechanisch bewegliche Teststrukturen durchgeführt. Deren Herstellung ist zeit- und kostenintensiv. Insbesondere die optischen Methoden, die auf den Schwingungseigenschaften des Festkörpers basieren, wurden eingesetzt, um die Folgen des Kristallwachstums zu untersuchen. Die Analyse der Schichtqualität kann somit rasch (taggleich) umgesetzt werden und erfordert keine aufwändige Probenpräparation, die bis zu 6 Monate in Anspruch genommen hat.

Basierend auf den theoretischen Untersuchungen der Strukturgeometrie von AlN wurden die Raman-Spektren von gesputterten AlN-Dünnfilmen hinsichtlich Peakverschiebungen und Verbreiterung rationalisiert, die direkt proportional zu den eingebauten Dehnungsfeldern sowie der Lebensdauer anisotroper Phononen sind. Die Ergebnisse der Raman- und FourierTransform-Infrarotspektroskopie wurden mithilfe der Röntgendiffraktometrie untersucht einem ultimativen Kristallographie-Werkzeug. Es wurde festgestellt, dass die Variationen der Dicken- und Dehnungsfelder über den 6-Zoll-Wafer während der Wachstumsoptimierung auf das tolerierbare Niveau reduziert sind. Basierend auf der Bestimmung der Kristallqualität wurden die Modelle entwickelt, die die anisotropen optischen Eigenschaften von AlN umfassen und häufig für spektroskopische Ellipsometrieuntersuchungen verwendet werden. Sie sind das schnellste Charakterisierungswerkzeug für Dünnfilme in der aktuellen Mikrotechnik. Insbesondere wurde die inhärente Eigenschaft des Kristallwachstums durch Magnetron-Sputtern, d.h. das säulenförmige Wachstum von AlN, modelliert, wodurch die Schätzung der Domänengrenzen auf der Grundlage der Ellipsometriemessungen ermöglicht wurde. Im Verlauf des E-PISA-Projekts wurde die Oberflächenmorphologie von AlNDünnfilmen durch Rasterkraftmikroskopie untersucht, um die Rolle von Sputterparametern für die Korngrößenverteilung zu bestimmen. Darüber hinaus wurde das gleiche Werkzeug verwendet, um die piezoelektrische Leistung der gesputterten AlN-Kristalle lokal zu untersuchen. Es wurde gezeigt, dass der Mittelwert des piezoelektrischen Koeffizienten außerhalb der Ebene sowohl von der Wachstumsmethode als auch vom Substrat der Wahl abhängt. 


\section{Die grundlegende Funktionsfähigkeit von AIN auf} Siliziumsubstrat wurde bei bis $1000{ }^{\circ} \mathrm{C}$ nachgewiesen.

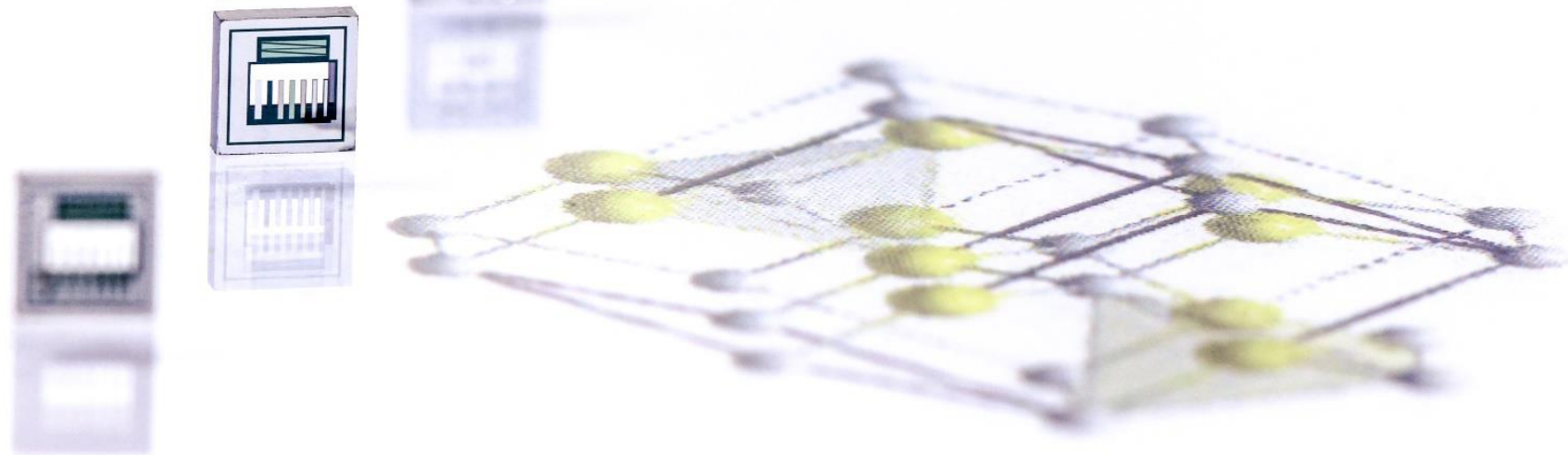


Der Effekt des Temperns nach dem Wachstum unter Umgebungsbedingungen wurde untersucht, um zu bestimmen, ob die Kristallqualität von AlN-Dünnfilmen erhöht werden kann, sobald die Kristalle gezüchtet sind. Wir haben gezeigt, dass die Optimierung der auf Si (111)Substrat gewachsenen Filme bei der Glasübergangstemperatur des Siliziumdioxids, die um $900^{\circ} \mathrm{C}$ wächst, begrenzt ist. Zusätzlich diffundieren die interstitiellen Sauerstoffatome, die in den für die MEMS-Herstellung verwendeten Si-Wafern vorhanden sind, durch das Si-Gitter zur Oberfläche, die am Wachstum der Si-Oxidschicht beteiligt ist. Schließlich beeinflusst eine solche Verwundbarkeit der AlN/Si-Grenzfläche die piezoelektrischen Eigenschaften der AlN-Schicht, die die Rekristallisation und die Bildung der nicht aktiven kubischen Körner zeigt.

Obwohl AlN ein sehr robustes Material ist, sind seine piezoelektrischen Koeffizienten niedriger als bei den meisten derzeit verwendeten piezoelektrischen Materialien. Um ihre Werte und damit die Empfindlichkeit und Amplituden der mechanischen Bewegung zu erhöhen, wird AlN mit verschiedenen Fremdatomen wie Scandium legiert, von denen gezeigt wurde, dass sie die piezoelektrische Leistung um das Vierfache verbessern. Solche AlScNLegierungen sind nicht leicht zu züchten, da die Komplexität des Phasendiagramms mit der Zugabe des dritten chemischen Elements erheblich zunimmt. Darüber hinaus bilden ScAtome überwiegend piezoelektrisch nicht aktive Steinsalznitridphasen. Um das Wachstum von AlScN zu optimieren, wurden Röntgenbeugungs- und optische Methoden angewendet, die zeigten, dass die Menge an Sc-Atomen, die durch Stickstoffplasma gesputtert wird, die Struktur der AlN-Dünnfilme dramatisch beeinflusst. Insbesondere zerstören die fremden ScAtome das Wurtzitgitter aufgrund ihrer größeren Größe und der Möglichkeit der sechsfachen Koordination. Ein solches Gitter der Legierung zeigt eine beträchtliche Erweichung, wie aus der Untersuchung der Phononeneigenschaften durch Raman-Spektroskopie sowie einer Erhöhung der Hochfrequenz-Dielektrizitätskonstante aufgrund der halbmetallischen Natur von $\mathrm{ScN}$ ersichtlich ist. Die Co-Bildung der kubischen Phasen wird bei dem relativ niedrigen Sc-Gehalt beobachtet, was die Verwendung solcher Legierungen inhärent einschränken kann. Trotzdem kann die effektive piezoelektrische Leistung diesen Mangel überwinden. Eine weitere Optimierung des Wachstums von AlScN-Legierungen ermöglicht eine genaue Abstimmung in Abhängigkeit von der erforderlichen Leistung sowie den verfügbaren Kosten. 


\section{Kohlenstoffnanoröhren \\ als \\ Grundlage \\ heterogener Systeme mit neuen Funktionalitäten.}

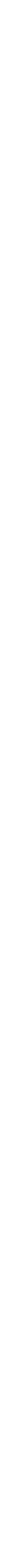




\section{Heterosystemintegration von CNT-Elektronik}

Um die Verstärkung kleinster Ladungsverschiebungen im AlN zu realisieren, müssen CNTFETs mit exzellenten Schaltverhalten und einer geringen Gatekapazität entwickelt werden. Dabei wird zusätzlich zur Typenseparation von CNTs, mit dem Ausschluss metallischer CNTs, ein CNT-Selbstassemblierungsprozess entwickelt.

Die durch die herstellungsbedingte Variation der CNT-Eigenschaften wie Länge, Durchmesser und Chiralität zieht auch eine Variation der elektronischen Eigenschaften und somit eine vergleichsweise flache Schaltkurve nach sich. Um ein exzellentes Schaltverhalten zu erreichen, wurde ein Kolonnen-Chromatographie-Aufbau etabliert, der eine Eingrenzung der Chiralitäts-/Durchmesserverteilung bis hin zur monochiralen Separation von CNTs mit geringen Durchmesser ermöglicht. Durch den bereits von Kataura ${ }^{1}$ beobachteten „Overloading“-Effekt, werden alle Adsorptionsplätze im Separationsmedium von der CNTSpezies mit der höchsten Wechselwirkung eingenommen. Dies erlaubt eine Schichtung von verschiedenen Spezies innerhalb des Chromatographiesystems. Durch sequentielles Ausspülen der adsorbierten CNTs in diesen Kolonnen können die unterschiedlichen Spezies nun separat extrahiert werden. Der realisierte Aufbau im Projekt E-PISA besteht aus 6 Kolonnen mit je $5 \mathrm{ml}$ Separationsmedium und ist darüber hinaus für die teilautomatisierte Prozessierung mit mikrofluidischen Ventilen zwischen den Kolonnen ausgestattet. Dies erlaubt die Extraktion von bis zu $10 \mathrm{ml}$ hochseparierten CNT-Dispersionen je Kolonne.

Die folgende Abbildung zeigt den realisierten Aufbau und die UV-Vis-NIR Absorptionsspektren von den separierten Dispersionen im Vergleich zur Originaldispersion. In den Spektren zeigt sich eine deutliche Signalerhöhung von Absorptionspeaks spezifischer CNT-Chiralitäten. Darüber hinaus sind in einer studentischen Projektarbeit weitere methodische Entwicklungen zur Nutzbarmachung der separierten Dispersionen für den ebenfalls im Projekt etablierten neuartigen Selbstassemblierungsprozess, welcher im folgenden Abschnitt erläutert wird.
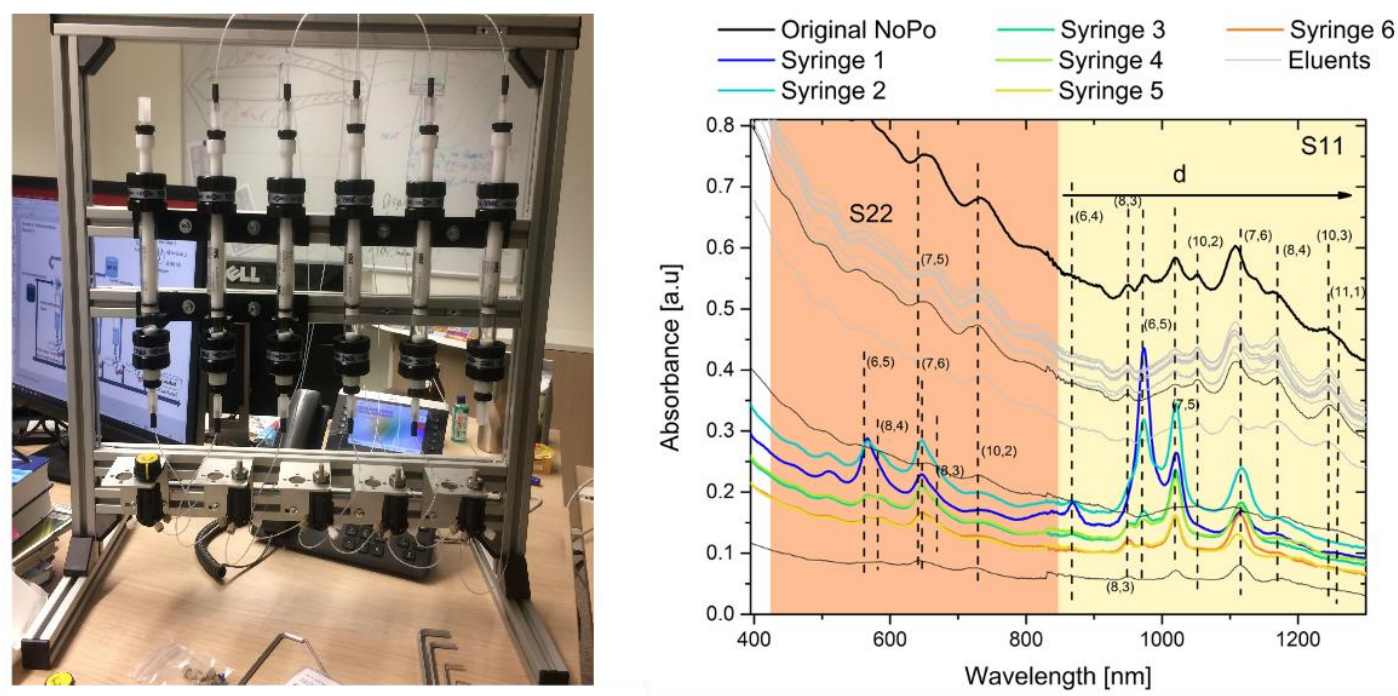

${ }^{1}$ Liu, H., Nishide, D., Tanaka, T. et al. Large-scale single-chirality separation of single-wall carbon nanotubes by simple gel chromatography. Nat. Comm. 2, 309 (2011). https://doi.org/10.1038/ncomms 1313 
Die derzeitige $200 \mathrm{~mm}$ wafer-level Prototypenfertigung von CNT-FETs erlaubt Ausbeuten von über $90 \%$ bei $300 \mathrm{~nm}$ Kanallänge und hoher Bauelementdichte.

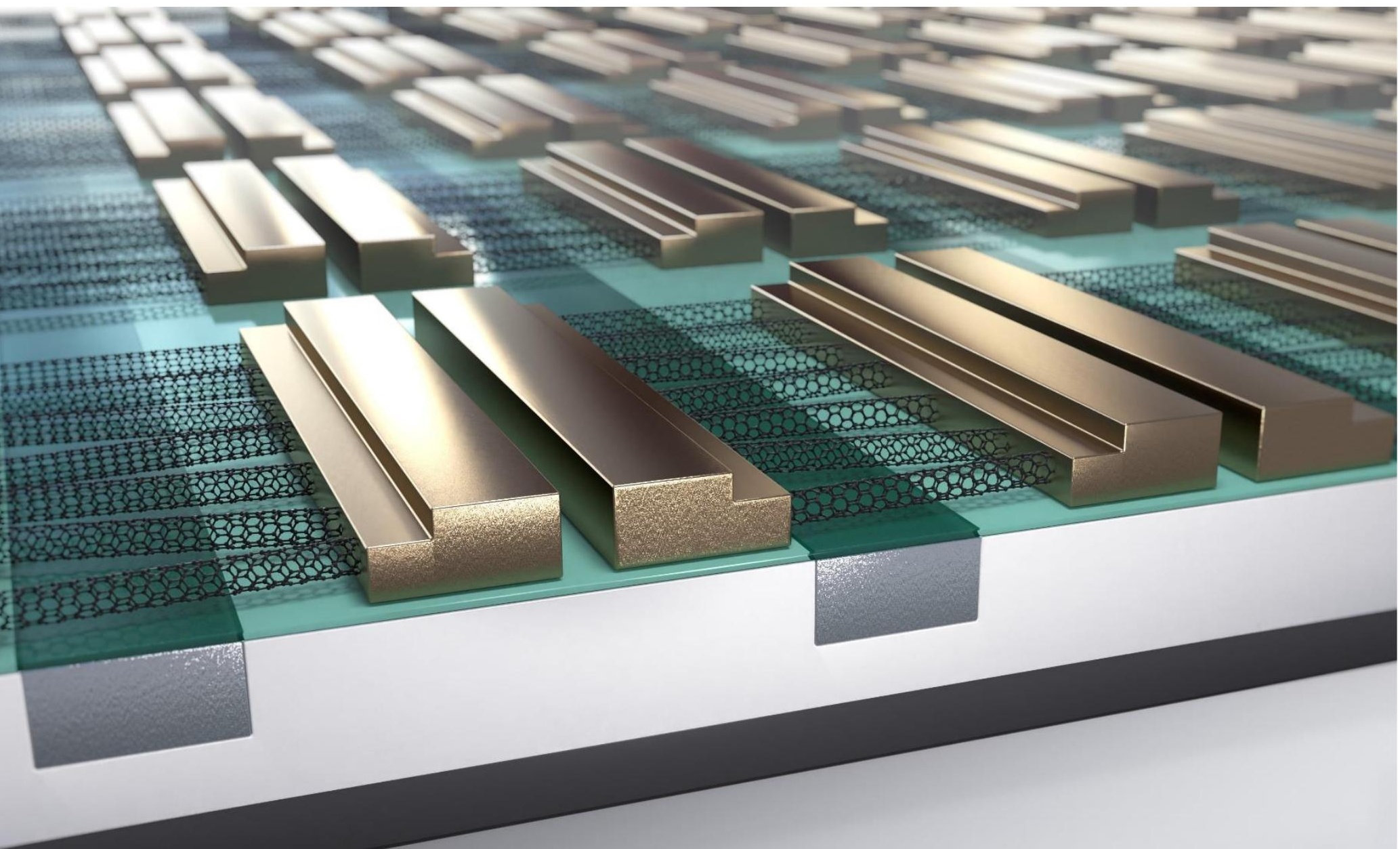


Einen Großteil der Projektarbeit floss in die Prozessentwicklung und Skalierung des CNTSelbstassemblierungsprozesses (CSA). Dabei bestand die größte Herausforderung in der Entwicklung einer hohen Prozessreproduzierbarkeit, da die extrinsischen Prozessparameter wie die Oberflächenenergie des Zielsubstrates, Dispersionseigenschaften, und -additiven immer herstellungsbedingten Schwankungen des Lieferanten unterliegen. Die größten Fortschritte hier wurden durch ein empirisch ermitteltes tiefgehendes Prozessverständnis der physikalisch und chemisch stattfindenden Grundprozesse und Abhängigkeiten, wie beispielsweise das Spreitverhalten des Chloroform-Dispersionsmediums auf Wasser in Abhängigkeit von dessen lagerungsbedingten Wassergehalts erreicht. Mittels definierter Vorbehandlung der Substrate, sowie einer Chloroformlagerung unter Inertgasatmosphäre konnten diese Schwankungen auf ein Minimum reduziert und somit die Reproduzierbarkeit signifikant erhöht werden.

Außerdem wurden in einer Vielzahl von systematischen Studien die intrinsischen Prozessparameter wie Oberflächenenergie des Zielsubstrates, Liftrate, Dispersionsflussrate, Substratabstand, und Anderen untersucht und optimiert. Im Projektverlauf konnten mit diesem Prozess können CNTs mit nahezu uniaxialer Ausrichtung und einer hohen Flächendichte der CNT-Assemblierung erreicht werden (siehe untenstehende Abbildung). Die erreichte Winkelverteilung des CNT-Films um die gewünschte Abscheideachse beträgt $\pm 18^{\circ}$.

Diese Prozessentwicklungen mündeten in der Wafer-level Demonstration von 10.000 individuell elektrisch kontaktierbaren CNT-FETs auf einem $200 \mathrm{~mm}$ Siliziumwafer mit globaler Si-Gate-Elektrode und ultradünnem high-k HfO2-Gatedielektrikum. Die hergestellten CNT-FETs zeichnen sich durch größtenteils exzellentem Schaltverhalten mit einem Subtreshold Slope von $150 \mathrm{mV} /$ Dekade, hoher Leitfähigkeit und hoher Transkonduktanz von bis zu $10 \mathrm{mS} / \mu \mathrm{m}$ Kanallänge aus. Dabei zeigt sich bereits eine erhöhte Reproduzierbarkeit.

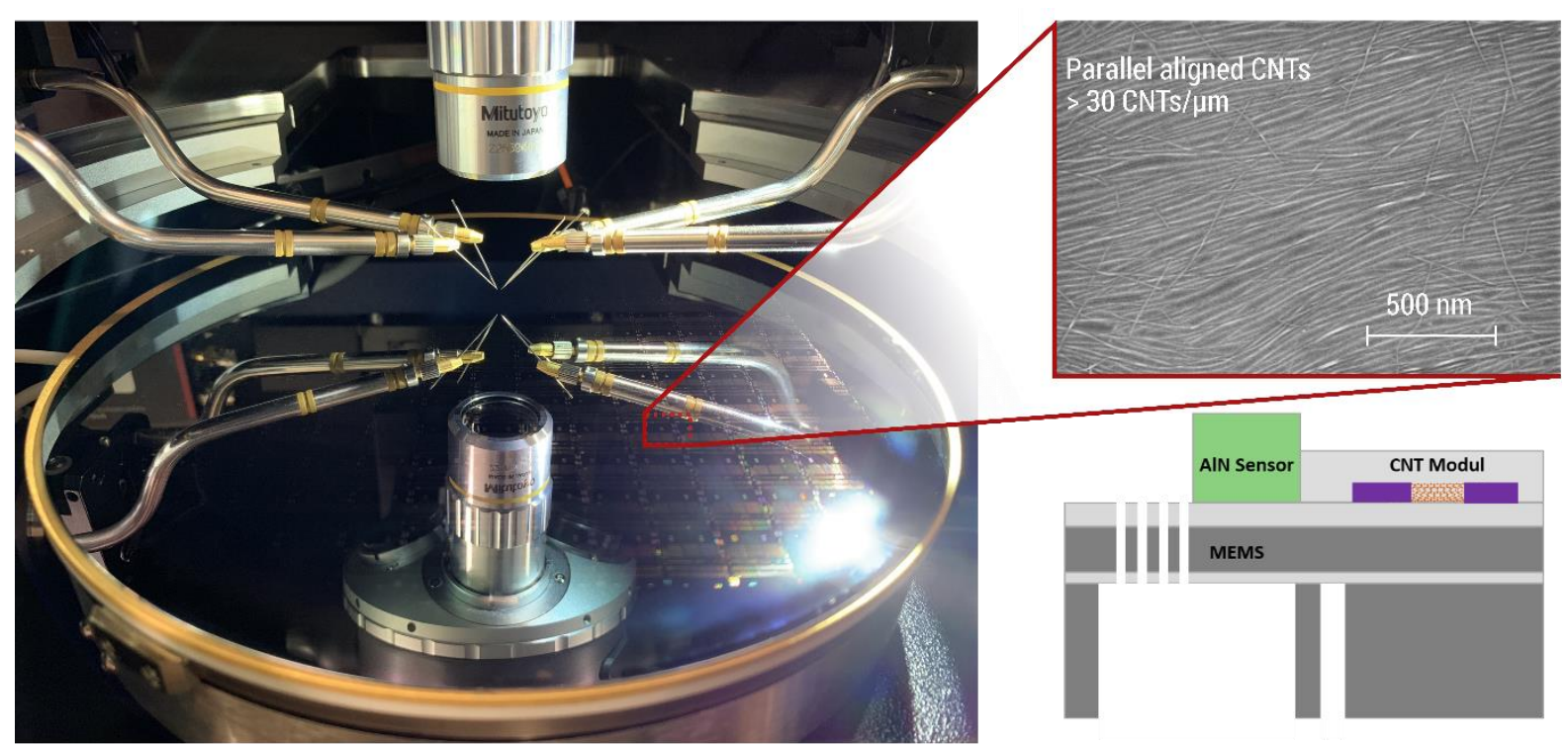


„...der Hauptvorteil [von industrieller Kooperation mit Forschungseinrichtungen] ist natürlich, dass man einen direkten Zugang zu Ressourcen, Capabilities aber auch zu Netzwerken der [akademischen] Projektpartner bekommt.“ 


\section{Innovationssysteme, Märkte und Forschungs- netzwerke}

In Arbeitspaket „Adaption und Diffusion“ wurde die Industrie und der Markt für MEMSTechnologie untersucht. Neben der Erhebung des Status Quo und der zukünftigen Potentiale der Technologie fokussierte sich die Forschung auf das Innovationssystem für MEMSTechnologie. Bei der Untersuchung des Innovationssystems wurden insbesondere die Förderlandschaften und Industrienetzwerke betrachtet.

Bei den Industrienetzwerken fokussierte die Erhebung das regionale Expertennetzwerk Silicon Saxony und das internationale Expertennetzwerk IVAM. Die gewählten Netzwerke dienten zudem als Zugang zu den Akteuren dieser Industrie und ermöglichten eine Befragung von Experten aus 12 Firmen, 6 KMU's und 6 Großunternehmen sowie von 2 Experten der Fraunhofer Gesellschaft für angewandte Forschung e.V. Die Interviews thematisierten den Status Quo und die Zukunft der Technologie, des Marktes und der Branche, Förderprogramme, Netzwerke, und Kooperationen.

Hinsichtlich der Einschätzung und Nutzung von Kooperation, Kollaboration und Netzwerken bestätigten insbesondere die KMUs Granovetters Theorie der "Schwachen Verbindungen" und Burts Konzept der "Strukturellen Löcher"2. Die Akteure nehmen keine Konkurrenz war, Sie sehen die Vorteile der Netzwerke und verbinden ihre eigenen kleinen, oft freundschaftlichen und regionalen Netzwerke mit größeren Netzwerken und überbrücken damit strukturelle Löcher, ermöglichen damit neue Informationsströme, Vorteile und Möglichkeiten für die eigene Organisation sowie potentiell das eigene kleine Netzwerk. Dieses beschriebene Verhalten insbesondere der KMUs ist im Einklang mit dem beschriebenen Verständnis der eigenen Organisation in der Industrie als konkurrenzloser Akteur aufgrund der eigenen Spezialisierung und eroberten Marktnische.

Die untersuchten formalen Netzwerke fungieren als Schnittstellen des Sektors und übernehmen die Rolle eines Brokers der die Organisationen verbindet und Möglichkeiten generiert, die den Aufbau von schwachen Verbindungen zwischen den Unternehmen fördern. Zudem aggregieren und verteilen sie branchenrelevante Informationen an ihre Mitglieder. Gefragt nach den Beweggründen für eine Partizipation an diesen Netzwerken, verwiesen die interviewten Unternehmen auf den Informations- und Technologieaustausch und die veranstalteten Events. Diese bieten Möglichkeiten zur Präsentation der eigenen Organisation und ihrer Produkte und Dienstleistungen. Die Veranstaltungen generieren Gelegenheiten zur Vernetzung mit anderen Organisationen - wie potentiellen Partnern, Kunden oder Zulieferern.

\footnotetext{
${ }^{2}$ Siehe Granovetter, M. (1973). The Strength of Weak Ties. American Journal of Sociology, 78(6), 1360-1380 und Burt, R. S. (1992): Structural Holes: The Social Structure of Competition, url: https://ssrn.com/abstract=1496205.
} 
„Es ist so, dass hier im Silicon Saxony alle Player der Wertschöpfungskette vorhanden sind. Man kann sich sowohl mit System- als auch mit Implementierungs-Fragestellungen auseinandersetzen."

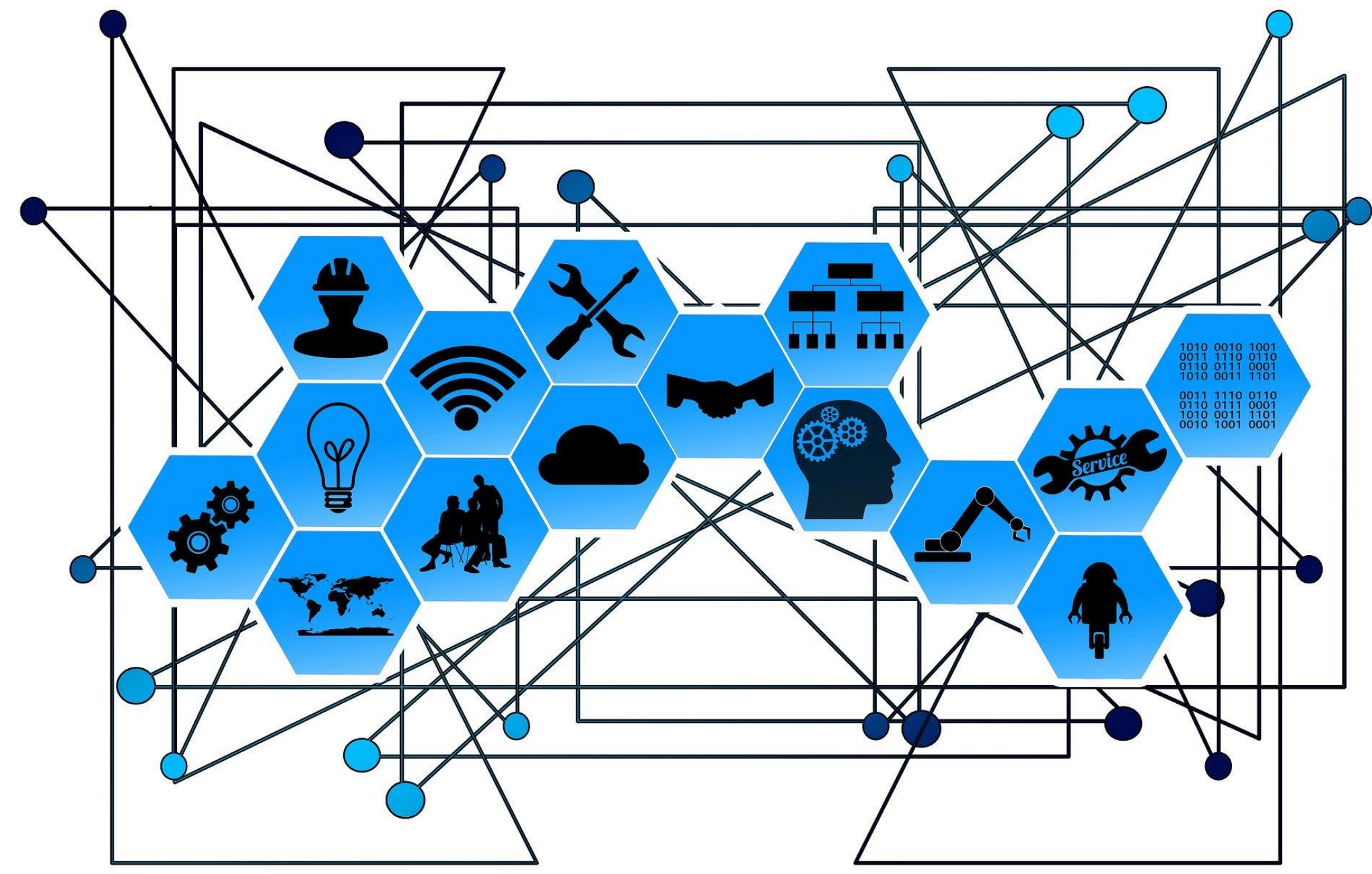


Alle befragten Unternehmen befürworteten Förderprogramme und gaben an, dass sie diese beobachten, selektieren und nutzen. Die KMUs betonten zudem die Risiken, insbesondere den finanziellen und bürokratischen Aufwand sowie die schwierige Vereinbarkeit mit Auftragsarbeiten bzw. Projekten für Kunden.

Die Organisationen argumentierten, dass es notwendig Kooperationen einzugehen, da sie oft nicht in der Lage sind, die notwendigen Produktionstechnologien und -kapazitäten allein bereitzustellen. Kooperation ermöglicht den Austausch von Wissen und Informationen, aber auch das teilen von materiellen Ressourcen wie Betriebsmitteln. Zusätzlich wird die Zusammenarbeit mit anderen Organisationen in Förderprogrammen sehr häufig vorausgesetzt.

Hinsichtlich der aktuellen Marktsituation betonten die Organisationen die Chancen in Verbindung mit der Digitalisierung im Allgemeinen, insbesondere der Industrie 4.0 und des Automotive Sektors. Da die MEMS-Industrie auf globaler Ebene miteinander verbunden und voneinander abhängig sind, besteht zusätzlich eine erhebliche Abhängigkeit und von der globalen Wirtschaft und Politik. Als zukünftige Trends wurde explizit der zukünftige Markt für medizinische MEMS-Anwendungen genannt und mit dem bereits etablierten AutomotiveMarkt verglichen. Der Automotive-Sektor wurde ebenfalls genannt, ebenso wie intelligente Mikrosysteme und NEMS.

Die Interviews bestätigten die Auffassung, dass MEMS eine disruptive Technologie war, die sich bereits etabliert und zu einer nachhaltigen Technologie entwickelt hat. Und als etablierte Technologie sind die MEMS Technologie und Markt ihrererseits anfällig für Disruption. Hinsichtlich potentieller Disruptionen verwiesen die Interviewpartner auf Alternativen zu Silizium als Basismaterial für Wafer, insbesondere für spezifische zukünftige Anwendungen. Explizit genannt wurden Silizium-Carbide und Gallium-Nitride als Basismaterialien und Umweltsensoren als Anwendungen. Zudem wurde die NEMS Technologie als potentielle Alternative zu MEMS genannt, die aufgrund der weiteren Miniaturisierung effizienter sind und insbesondere bei bestimmten Anwendungen klare Vorteile gegenüber MEMS haben. Explizit genannt wurden hierbei Anwendungen im Bereich der RF (Radio Frequency) Technologie. Zwei Firmen gaben an, dass sie Anfragen für Lösungen auf Basis von NEMSTechnologie erhalten, aber nur eine KMU erklärte, dass sie diese Lösungen auch anbieten. Die Großunternehmen erklärten, dass sie sich auf MEMS fokussieren und keinen Wechsel von MEMS zu NEMS erwägen.

Ergebnisse der Untersuchung legen nahe, dass die Produktion, Diffusion und Nutzung von Wissen und Netzwerken wichtige Funktionen von Innovationssystemen sind und das insbesondere Netzwerke weiter untersucht werden sollten. Die Auswertung der Interviews ergab deutliche Unterschiede bei der Wahrnehmung und Nutzung von formellen und informellen Netzwerken, und von internationalen und regionalen Netzwerken. 
„Auf der Chancen-Seite ist für uns ganz klar der Biomedical-Sektor [...] dort sehen wir für die MEMS Potential. [...] Man könnte auch sagen, Medical ist das neue Automotive." 
Entsprechend wird empfohlen, diese Unterscheidungen in weiterführende Untersuchungen von Netzwerken zu berücksichtigen. Auch eine Unterscheidung der Akteure nach Typen (wie MNUs, KMU, öffentliche Einrichtungen und Vereine), ihrer Rollen und Strategien in den Netzwerken und wie diese sich auf die anderen Akteure im Netzwerk auswirkt, könnte weitere interessante Einsichten generieren. Darüber hinaus legen die Ergebnisse zudem nah, dass weiterführende Forschung zu organisationalen Förderungs-, Netzwerk- Kollaborationsund Kooperationsstrategien angebracht ist. In dieser Untersuchung reichte das beschriebene Organisationsverhalten vom ,Fokus auf Kooperation und Kollaboration', über , gemeinsames Wachsen" bis hin zu, in den Worten des Interviewten Experten, ,opportunistischer Netzwerkstrategie', der Konzentration auf die eigenen Vorteile und Gewinne.

Hinsichtlich des Marktes und Sektors für MEMS Technologie deuten die gesammelten und ausgewerteten Daten an, dass weiterführende Untersuchungen zum disruptiven Potential von alternativen Wafer-Technologien für spezifische Anwendungen wie Umweltsensoren sowie der NEMS Technologie, insbesondere hinsichtlich spezieller Anwendungsgebiete (oder Nischen), in denen NEMS besser funktionieren sollten als MEMS, wie bei beispielsweise RF Sensoren. 
Exakte Modelle zur Zuverlässigkeit mechanisch und elektrisch belasteter Mikro-Bauelemente sind ein Tor zur industriellen Einführung neuer Mikrosysteme in die industrielle Anwendung. 


\section{Zuverlässigkeit piezoelektrischer Schicht- systeme der Mikrotechnologie}

Mit der schnellen und fortschreitenden Evolution von MEMS-Strukturen müssen sich Wissenschaftler ständig neuen Herausforderungen stellen. Hierzu sind neue Materialsysteme und unterschiedliche physikalische Konzepte notwendig. Die Verwendung neuer Materialien und Prozesstechnologien (die wiederum zu neuen Materialeigenschaften führen) bedingen zunächst eine größere Unkenntnis der Zuverlässigkeit dieser neuen MEMS-Strukturen.

Bei der Verwendung von Aluminiumnitrid für piezoelektrische Aktor- und Sensorsysteme sind komplett neuartige Schichtaufbauten und Materialsysteme notwendig. Zur Abschätzung der Lebensdauer und des Materialverhaltens während des Betriebes der Strukturen ist daher ein tiefgehendes Verständnis der Materialtheorie und der Schichtinteraktionen notwendig.

Im Rahmen des E-PISA-Projektes bestand hierbei die Möglichkeit der Prozessierung spezieller Materialstrukturen, um die Materialeigenschaften der einzelnen Schichten zu charakterisieren. Hierbei erfolgte zum einen die Analytik durch elektronenmikroskopische Untersuchungen, etwa zur Untersuchung von Materialdiffusion. Außerdem wurden Nanoindenter-Experimente durchgeführt mit das elastisch-plastische Verhalten der neuen Materialien zu bestimmen.

Die so bestimmten Materialeigenschaften waren Ausgangspunkt für die Erstellung von finiteElemente-Simulationen. Diese wurden auf die Vielzahl der verwendeten Geometrien und unterschiedliche Materialsysteme angewendet und dienten dazu das realisierbare Schwingungsverhalten und verschiedene Aspekte der Zuverlässigkeit. Hier konnte gezeigt werden, dass die verwendeten Systeme bei entsprechend hohen Amplituden zu einer Ermüdung der oberen Aluminiumschicht in Folge starker plastischer Verformungen führen kann.

Im Rahmen des Projektes wurden zusätzlich Ermüdungsexperimente durchgeführt, die die theoretischen Vorhersagen validieren konnten. Der Betrieb der Schwingungsstrukturen führt im vorhergesagten Bereich zu Schäden in der oberen Elektrode, die sich in einer starken Vergröberung der Oberflächenrauigkeit äußern. Diese Rauigkeit wurde mittels Rasterkraftmikroskop gemessen und statistisch ausgewertet. Hierbei konnten im Bereich der Ermüdung deutlich größere kornartige Strukturen vermessen werden als in unbeschädigten Bereichen. Zusätzlich konnte der Schaden optisch durch eine Verdunklung des entsprechenden Bereichs detektiert werden.

Im Projektrahmen war es zusammenfassend möglich Struktur-Eigenschafts-Beziehungen der Materialsysteme $\mathrm{zu}$ bestimmen und daraus ein theoretisches Modell zu erstellen, um bestimmte Design-Richtlinien zu definieren und das Verhalten der MEMS-Strukturen vorherzusagen. Diese Modelle konnten zusätzlich durch experimentelle Methoden bestätigt werden und dienen der Definition von zukünftigen, ermüdungsfesten Strukturen. 



\title{
Veröffentlichungen und Auszeichnungen
}

\author{
Y. M. Azhniuk, D. Solonenko, V. Y. Loya, V. Kryshenik, V. Lopushansky, A. \\ Mukherjee, A. Gomonnai, and D. Zahn. Flexoelectric and local heating effects on CdSe \\ nanocrystals in amorphous As2 Se3 films. Materials Research Express, \\ 6(9):095913, 2019
}

Y. M. Azhniuk, V. Dzhagan, D. Solonenko, V. Loya, I. Grytsyshche, V. Lopushansky, A. Gomonnai, and D. R. Zahn. In-doped As2Se3 thin films studied by Raman and X-ray photoelectron spectroscopies. Applied Surface Science, 471:943-949, 2019

D. Solonenko, O. Selyshchev, D. R. Zahn, and P. Vogt. Oxidation of epitaxial silicene on Ag (111). physica status solidi (b), 256(2):1800432, 2019

Y. M. Azhniuk, D. Solonenko, E. Sheremet, V. Dzhagan, V. Y. Loya, I. Grytsyshche, S. Schulze, M. Hietschold, A. Gomonnai, and D. Zahn. Structural and optical study of Zndoped As2 Se3 thin films: Evidence for photoinduced formation of ZnSe nanocrystallites. AIP Advances, 9(6):065212, 2019

Y. M. Azhniuk, D. Solonenko, V. M. Dzhagan, A. Mukherjee, V. Y. Loya, I. V. Grytsyshche, V. V. Lopushansky, A. V. Gomonnai, and D. R. Zahn. Laser-induced formation of $\mathrm{CdS}$ crystallites in Cd-doped amorphous arsenic sulfide thin films. physica status solidi (b), 256(2):1800298, 2019

Y. M. Azhniuk, D. Solonenko, V. Loya, I. Grytsyshche, V. Lopushansky, A. V. Gomonnai, and D. R. Zahn. Raman evidence for surface oxidation of amorphous As2 S3 thin films under ultraviolet irradiation. Applied Surface Science, 467:119-123, 2019

S. Böttger et al - Scaling up Integration of Carbon Nanotubes into Micro-ElectroMechanical Systems, Conf. Proceedings Smart Systems Integration 2018 SSI, Dresden

S. Böttger, J. Bonitz, M. Kini, S.E. Schulz, S. Hermann - Adding CNT-based functionality to MEMS: A technology demonstration for strain and IR sensors on wafer scale, Conf. Proceedings Mikro-Nano-Integration 2018 MNI, Dortmund

S. Böttger, J. Bonitz, F. Al-Falahi, S.E. Schulz, S. Hermann et al. - Functional carbon nanotubes for MEMS applications: Miniaturized strain sensor \& black coating for infrared devices. MikroSystemTechnik Kongress, Berlin, Germany, 2019, pp. 1-4.

S. Böttger, C. Wagner, F. Lorkowski, M. Hartmann, J. Schuster, S. Hermann - Sensitivity control of carbon nanotube-based piezoresistiv sensors by drain-induced barrier thinning. Sensors and Actuators A 295:288-295, 2019

S. Böttger, C. Wagner, F. Lorkowski, M. Hartmann, G. Heldt, D. Reuter, J. Schuster, S. Hermann - Role of contacts in carbon nanotube giant piezoresistive sensors, 20th International Conference on Solid-State Sensors, Actuators and Microsystems \& Eurosensors XXXIII (TRANSDUCERS \& EUROSENSORS XXXIII), Berlin, Germany, 2019, pp. 2400-2403 


\section{Outstanding Paper Award Winner auf der}

\section{Transducer 2019}

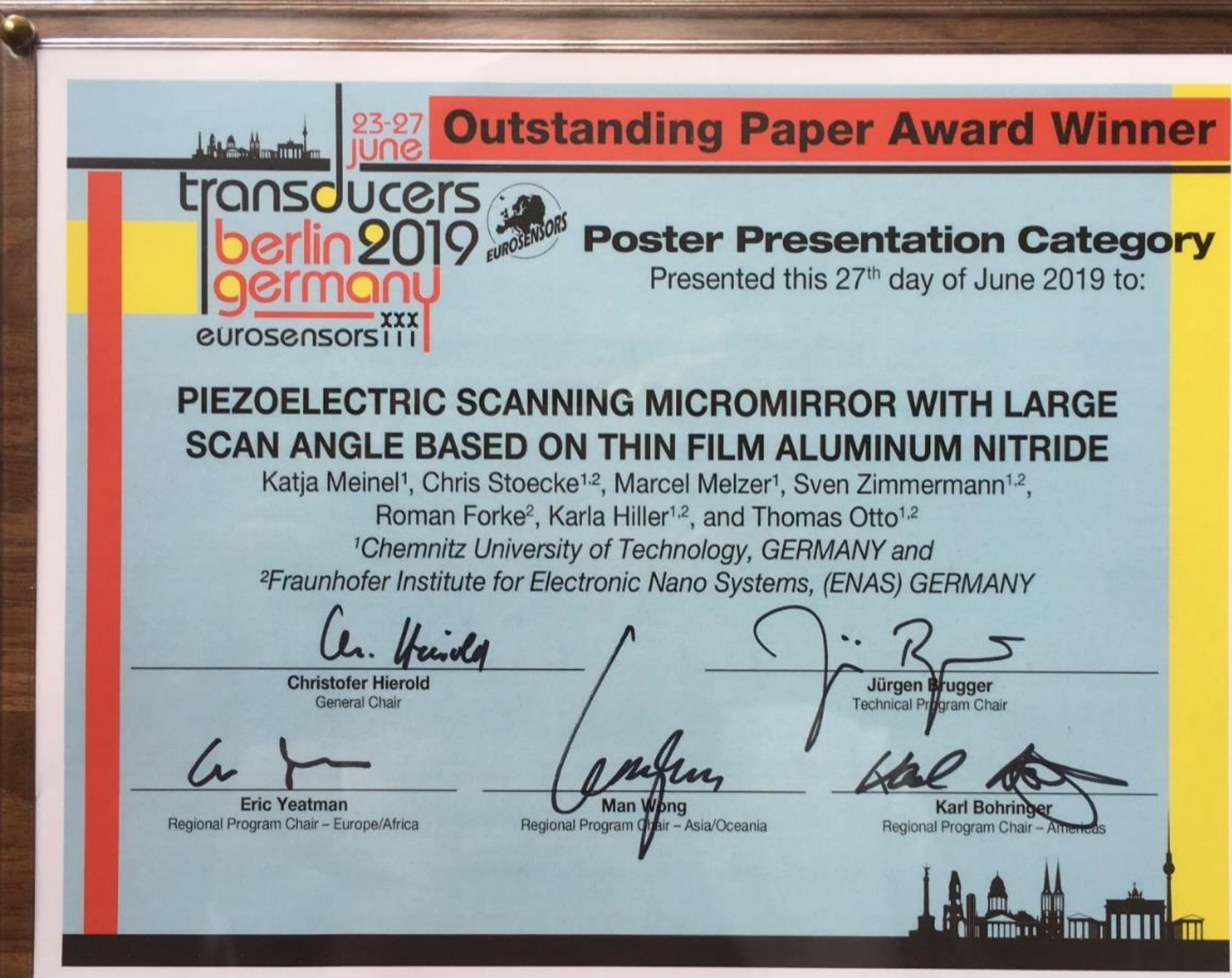


J. Bucher and J. P. Schmitten. How visionaries and narratives explicate possible futures and create paths from imagination to innovation. Exploring the Fiction-Innovation-Nexus looking at the narratives, manifestation and discourses of MEMS, the Hyperloop and blockchain technologies. New Economic Sociology NES Workshop 2019, 20.21.05.2019, Warsaw

J. Bucher and S. Hüsig. Innovation as Manifesting Imagination: Exploring the Role of Imaginations and Imaginators at the Front-End of Innovation, R\&D Management Conference 2019, 17.-21.06.2019, Paris, France

J. Bucher and S. Hüsig. Conceptualizing Imagination as an Inherent Element of the Innovation Process. ArtemOCC 2020, 19.-21.03.2020, Chemnitz, Book of Abstracts, ARTEM OCC 2020 Edition, 162-165.

J. Bucher and A. Weller. From the Visual Turn to Turned Up Visuality: Modes of Interaction in the Digitalized Era and Ways to Utilize Them. In: Fields, Z., Bucher, J. \& Weller, A. (Eds., 2019): Imagination, Creativity, and Responsible Management in the Fourth Industrial Revolution, IGI Global.

A. Dressler and J. Bucher. Introducing a sustainability evaluation framework based on the Sustainable Development Goals applied to four cases of South African Frugal Innovation. Special Issue: The role of SDGs for progressing sustainability, Business Strategy and Development, 2018, 276-285, https://doi.org/10.1002/bsd2.37.

M. Ezawa, E. Salomon, P. De Padova, D. Solonenko, P. Vogt, M. Dvila, A. Molle, T. Angot, and G. Le Lay. Fundamentals and functionalities of silicene, germanene, and stanene. La Rivista del Nuovo Cimento, 41:175, 2018

Z. Fields, J. Bucher and A. Weller: Preface. In: Fields, Z., Bucher, J. \& Weller, A. (Eds.): Imagination, Creativity, and Responsible Management in the Fourth Industrial Revolution, 2019, IGI Global.

Z. Fields, J. Bucher and A. Weller (Eds.): Imagination, Creativity, and Responsible Management in the Fourth Industrial Revolution, 2019, IGI Global.

R. Frenz, J. Bucher and A. Hermann-Fankhänel. Is an Artist a Better Scientist? An Empirical Analysis on the Impact That Artistic Activity Has on a Scientist's Achievement. In: Fields, Z., Bucher, J. \& Weller, A. (Eds., 2019): Imagination, Creativity, and Responsible Management in the Fourth Industrial Revolution, IGI Global.

M. Hartmann, J. Tittmann-Otto, S. Böttger, G. Heldt, M. Claus, S.E. Schulz, M. Schröter, S. Hermann - Gate Spacer Investigation for Improving the Speed of High-Frequency Carbon Nanotube-Based Field-Effect Transistors ACS APpl. Mater. Interfaces 2020, 12, 27461-27466

D. Heinbucher and J. Bucher. How do shared mental models foster team creativity? A team level analysis of drivers of ideation in the team creativity process of innovation teams. 3rd ARTEM Organizational Creativity and Sustainability International Conference, 19.-21.03.2020, Chemnitz, Book of Abstracts, ARTEM OCC 2020 Edition, 75-77. 


\section{Die Arbeiten der Nachwuchsforschergruppe} beflügeln Forschung und Entwicklung vom sächsischen KMU bis zum Global Player. 
C. Huber and J. Bucher: Drink the Jungle: Business shamans and their unconventional coaching methods. 3rd ARTEM Organizational Creativity and Sustainability International Conference,19.-21.03.2020, Chemnitz, Book of Abstracts, ARTEM OCC 2020 Edition, 170-173.

D. Kriebel and J. E. Mehner, "An Accurate Method for Automated Reduced Order Modelling of Frequency Depending Fluid-Structure Coupling Effects in Modal Subspace," 2019 Symposium on Design, Test, Integration \& Packaging of MEMS and MOEMS (DTIP), Paris, France, 2019, pp. 1-6.

D. Kriebel et al., "Design Automation for Micro-Electro-Mechanical Systems," 2018 International Semiconductor Conference (CAS), Sinaia, 2018, pp. 43-50.

D. Kriebel, H. Schmidt, M. Freitag and J. E. Mehner, "Automated reduced order model generation for MEMS," 2018 Symposium on Design, Test, Integration \& Packaging of MEMS and MOEMS (DTIP), Roma, 2018, pp. 1-6.

G. Le Lay, D. Solonenko, and P. Vogt. Synthesis of silicene. In Silicene, pages 99-113. Springer, Cham, 2018.

K. Meinel, C. Stöckel, M. Melzer, S. Zimmermann, R. Forke, K. Hiller, T. Otto: "Piezoelectric scanning micromirror with built-in sensors based on thin film aluminum nitride," 2019 IEEE SENSORS, Montreal, QC, Canada, 2019, pp. 1-4.

K. Meinel, C. Stöckel, M. Melzer, S. Zimmermann, R. Forke, K. Hiller, T. Otto: Outstanding Paper Award, TRANSDUCERS \& EUROSENSORS XXXIII, Berlin, Germany, 2019, June 27.

K. Meinel, C. Stöckel, M. Melzer, S. Zimmermann, R. Forke, K. Hiller, T. Otto, "Piezoelectric scanning micromirror with built-in sensors based on thin film aluminum nitride," in IEEE Sensors Journal, 2020, doi: 10.1109/JSEN.2020.2997873.

J. Molina, J. Bucher, S. Speelman \& A. Lange. Understanding frugal innovations in the water sector and their impact on sustainable development: insights from two social innovations in México. 3rd ARTEM Organizational Creativity and Sustainability International Conference, 19.-21.03.2020, Chemnitz, Book of Abstracts, ARTEM OCC 2020 Edition, 95-98.

J. Molina-Maturano, J. Bucher and S. Speelman. Understanding and Evaluating the Sustainability of Frugal Water Innovations in México: An Exploratory Case Study. Journal of Cleaner Production (2020), https://doi.org/10.1016/j.jclepro.2020.122692

D. Solonenko et al - Chemical Properties of Silicon in 2D and quasi-1D Epitaxial Structures, International conference "Flatlands Beyond Graphene-2018, Leipzig, 2018, p. 18

D. Solonenko, V. Dzhagan, S. Cahangirov, C. Bacaksiz, H. Sahin, D. R. Zahn, and P. Vogt. Hydrogen-induced sp2 - sp3 rehybridization in epitaxial silicene. Physical Review B, 96(23):235423, 2017 
D. Solonenko, J. Gasiorowski, D. Apaydin, K. Oppelt, M. Nuss, W. Keawsongsaeng, G. Salvan, K. Hingerl, N. S. Sariciftci, D. R. Zahn, et al. Doping-induced polaron formation and solid-state polymerization in benzoporphyrin-oligothiophene conjugated systems. The Journal of Physical Chemistry C, 121(44):24397-24407, 2017

D. Solonenko, O. D. Gordan, G. Le Lay, D. R. Zahn, and P. Vogt. Comprehensive Raman study of epitaxial silicene-related phases on $\mathrm{Ag}$ (111). Beilstein journal of nanotechnology, 8(1):1357-1365, 2017

D. Solonenko and P. Vogt. Epitaxial silicene. In 21st Century Nanoscience - a Handbook, page n/a. CRC Press, 2020

D. Solonenko, C. Schmidt, C. Stoeckel, K. Hiller, and D. R. Zahn. The limits of the postgrowth optimization of AlN thin films grown on $\mathrm{Si}$ (111) via magnetron sputtering. physica status solidi (b), page 1900400, 2019

O. Stroyuk, A. Raevskaya, F. Spranger, O. Selyshchev, V. Dzhagan, D. Solonenko, N. Gaponik, D. R. Zahn, and A. Eychmüller. Mercury-indium-sulfide nanocrystals: A new member of the family of ternary in based chalcogenides. The Journal of Chemical Physics, 151(14):144701, 2019

P. Thoma, M. Monecke, O.-M. Buja, D. Solonenko, R. Dudric, O.-T. Ciubotariu, M. Albrecht, I. G. Deac, R. Tetean, D. R. Zahn, et al. Polycrystalline La1-x SrxMnO3 films on silicon: Influence of post-deposition annealing on structural, magneto-optical, and magnetoelectrical properties. Applied Surface Science, 427:533-540, 2018

C. Wagner, T. Blaudeck, P. Meszmer, S. Böttger, F. Fuchs, S. Hermann, J. Schuster, B. Wunderle, S.E. Schulz - Carbon Nanotubes for Mechanical Sensor Applications. physica status solidi (a), 216:19, 2019, p. 1900584 


\section{.}

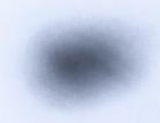

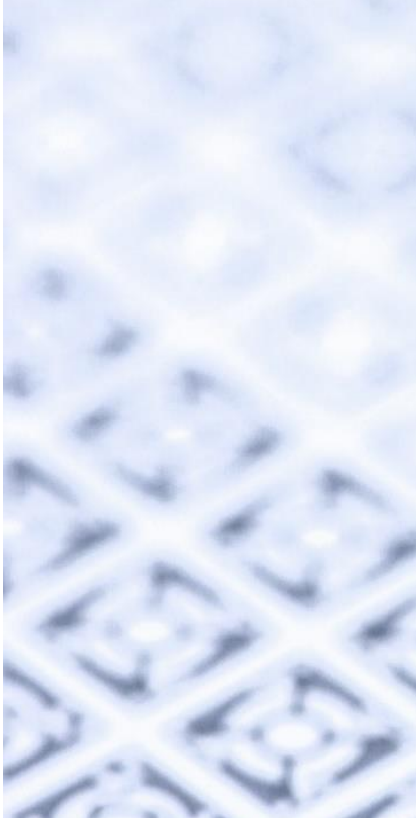

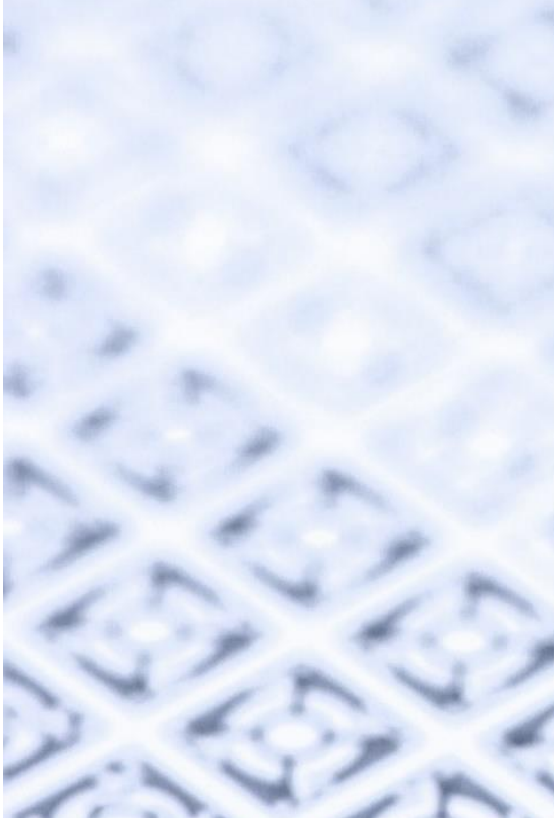

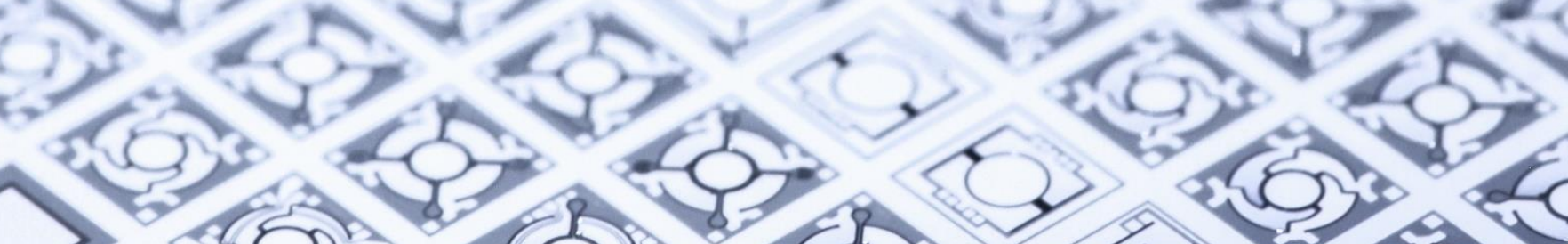

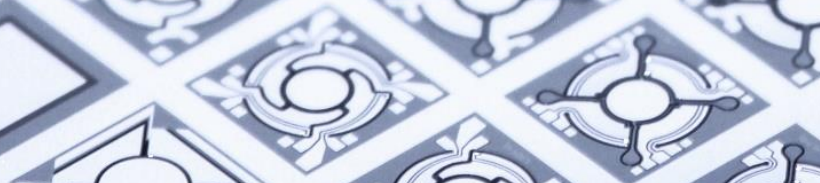

2

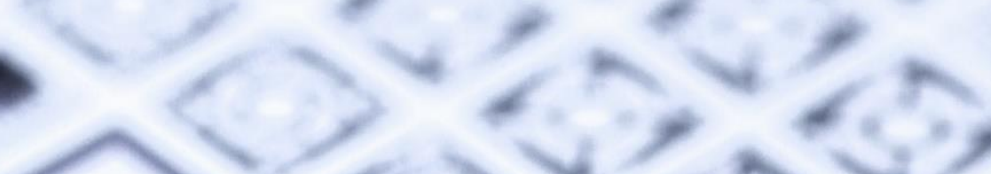

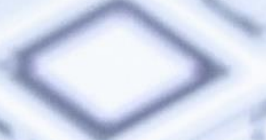

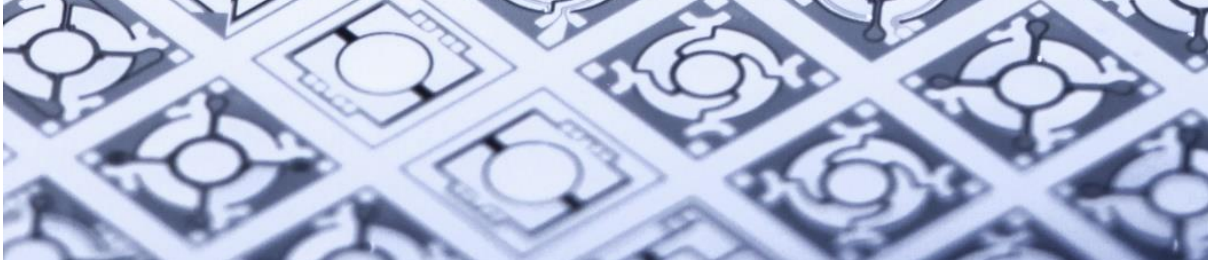

-D V

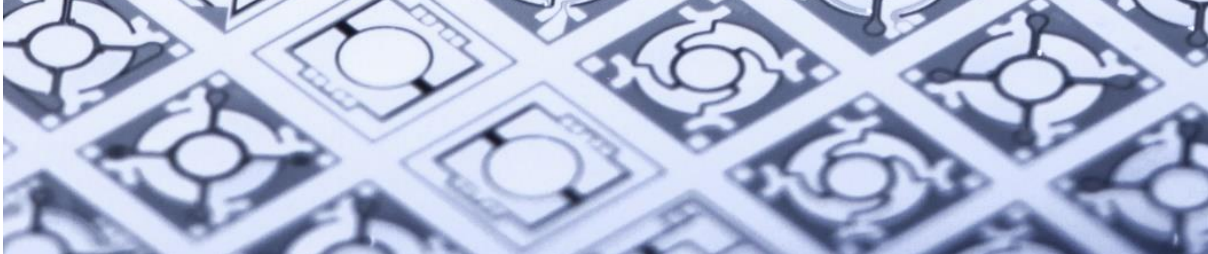
$43=2530$ ती (i) $ख 5 ?$ 


\section{Zukunftsfähigkeit}

Die Zukunftsfähigkeit von technologischen Innovationen kann unter anderem an der Verwertung im Projekt bearbeiteten Themen gemessen werden. Als Universität gibt es drei Möglichkeiten der Verwertung. Grundlagenorientierte Technologien und Applikationen müssen innerhalb der akademischen Forschung tiefergehend untersucht werden. Für industriell anwendungsfähige Themen ist eine direkte industrielle Verwertung (in der Industrie drittmittelgeförderte Projekte) geeignet. Für anwendungsnahe Entwicklungen eignen sich öffentlich geförderte Kooperationsprojekte mit Industriebeteiligung. Die Forschungsergebnisse der Nachwuchsforschergruppe haben in alle drei Bereichen, universitäre Forschung, anwendungsorientierte und öffentliche Entwicklung mit Industriekooperation, sowie reine Industriekooperationen starke Impulse gegeben.

Die vom ZfM (Zentrum für Mikrotechnologien der TU Chemnitz) bereitgestellte und u. A. in der Nachwuchsforschergruppe weiterentwickelte AlN-Technologie wird in enger Kooperation mit dem Fraunhofer ENAS mit Fokus auf industrielle Verwertung weiterentwickelt. Die Kooperation mit der Fraunhofer Gesellschaft eignet sich im Besonderen, um neu gewonnener Erkenntnisse gezielt in der Industrie zu verwerten. Nachfolgend ist eine Auswahl der akademischen und industriellen Anfragen zu in der Nachwuchsforschergruppe entstandenen Technologien und Applikationen aufgelistet (siehe hierzu auch die Abbildung auf S.6 in diesem Bericht), die häufig über die Kooperation mit der Fraunhofer Gesellschaft synergetisch generiert wurden.

Das ZfM hat auf Basis der Ergebnisse der NFG eine For-Mikro Förderung des BMBF zur Weiterentwicklung piezoelektrischer Wake-Up Funktionalitäten für energieautarke Sensorik in der Industrie 4.0 gewonnen. Im Konsortium sind über zehn Firmen aus der Industrie vertreten.

Die Ergebnisse der Wake-Up MEMS haben auch im Bereich Medizintechnik bei der Firma Biotronik Aufmerksamkeit erregt. Die Nachwuchsforscher konnten am Fraunhofer ENAS eine erste direkt beauftragte Studie zur Machbarkeit von Wake-Up Systemen in Implantaten umsetzen.

Dem Fraunhofer ENAS und dem ZfM gelang es so mit Hilfe der vorhandenen AlN Technologie einen Großauftrag der Firma Panasonic zu erhalten, bei dem AlN die Schlüsseltechnologie zur Problemlösung ist. Das ZfM ist Unterauftragnehmer in dem Projekt.

Ein Konsortium von Fraunhofer und Universitäten nutzt die AlN-Technologie, um in einem Transferprojekt AlN Technologie für piezoelektrische Ultraschallwandler zu nutzen. Neben der TU Chemnitz und TU Dresden ist auch das Fraunhofer Konsortialpartner.

Die Entwicklungen im Bereich der AIN MOEMS haben die Entwicklung von MEMS in der Plasmadiagnostik inspiriert. Innerhalb einer DFG geförderten Sachbeihilfe wird die Machbarkeit von der TU Chemnitz untersucht.

Die Ergebnisse bei den endoskopischen 2D MOEMS haben bei den Forschern der klinischen Diagnostik der TU Dresden Interesse geweckt. Eine Kooperation wird aufgebaut. 


\section{Danksagung}

Die Teammitglieder der Nachwuchsforschergruppe bedanken sich herzlichst bei den beteiligten ProfessorInnen der TU Chemnitz, die dieses Projekt durch ihre nicht vergüteten Leistungen bei der Beantragung, der Unterstützung bei den Forschungsaktivitäten, der Betreuung der Qualifizierungsarbeiten und der Publikation der Forschungsergebnisse erst ermöglicht haben. Wir danken den zahlreichen beteiligten TechnikerInnen und Hilfskräften, die uns in unseren Forschungsprojekten unterstützt haben. Dank gilt zudem Ina Schatz, die uns bei der Berichterstattung, Organisation und vor allem der formalen Verwaltung des Projektes unterstützt hat. Weiterhin danken wir dem Freistaat Sachsen, insbesondere der Sächsischen Aufbaubank, sowie der Europäischen Union, insbesondere dem Europäischen Sozialfonds, für die Förderung des E-PISA-Projektes. 


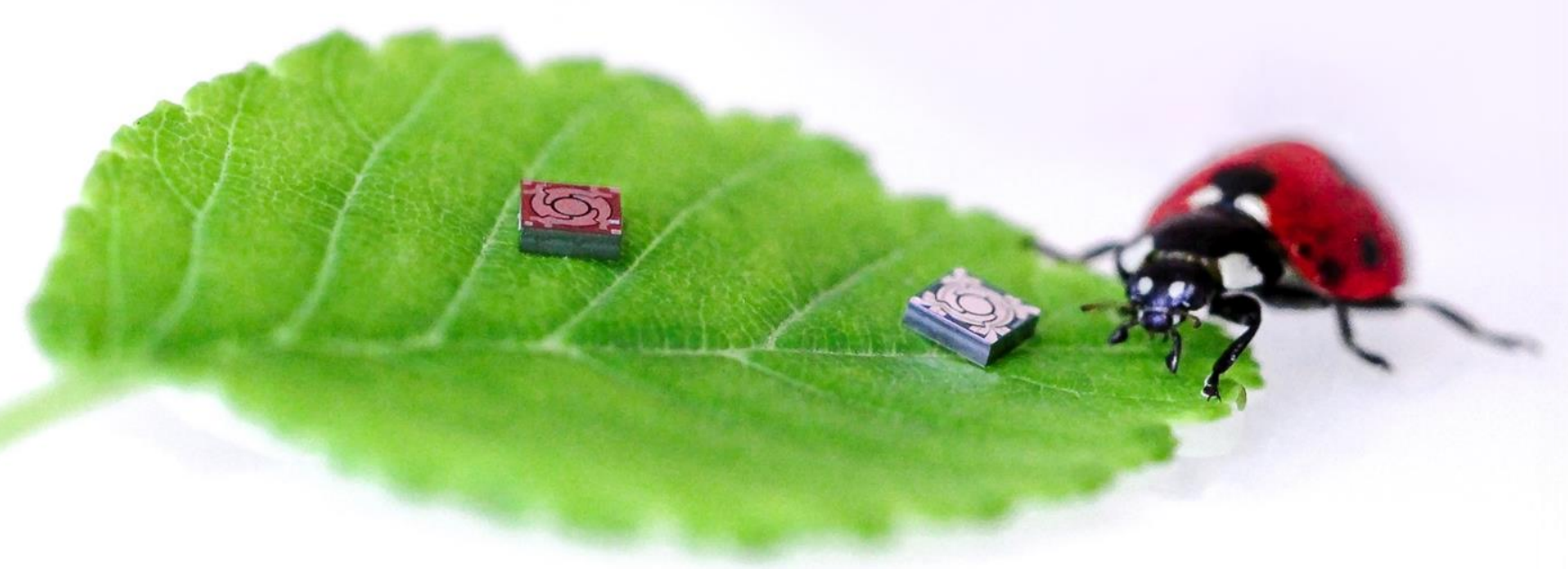

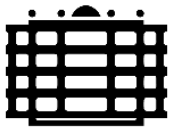

TECHNISCHE UNIVERSITÄT

CHEMNITZ
TU Chemnitz

Dr. Chris Stöckel, Dr. Julien Bucher

Chris.stoeckel@zfm.tu-chemnitz.de

www.tu-chemnitz.de 